\title{
The Influence of Stratification and Nonlocal Turbulent Production on Estuarine Turbulence: An Assessment of Turbulence Closure with Field Observations
}

\author{
MALCOLM E. SCULLY \\ Center for Coastal Physical Oceanography, Department of Earth and Atmospheric Science, Old Dominion \\ University, Norfolk, Virginia \\ W. Rocky Geyer AND JOHn H. TROWBRIDGE \\ Woods Hole Oceanographic Institution, Woods Hole, Massachusetts
}

(Manuscript received 18 March 2010, in final form 11 August 2010)

\begin{abstract}
Field observations of turbulent kinetic energy (TKE), dissipation rate $\varepsilon$, and turbulent length scale demonstrate the impact of both density stratification and nonlocal turbulent production on turbulent momentum flux. The data were collected in a highly stratified salt wedge estuary using the Mobile Array for Sensing Turbulence (MAST). Estimates of the dominant length scale of turbulent motions obtained from the vertical velocity spectra provide field confirmation of the theoretical limitation imposed by either the distance to the boundary or the Ozmidov scale, whichever is smaller. Under boundary-limited conditions, anisotropy generally increases with increasing shear and decreased distance to the boundary. Under Ozmidov-limited conditions, anisotropy increases rapidly when the gradient Richardson number exceeds 0.25 . Both boundary-limited and Ozmidov-limited conditions demonstrate significant deviations from a local production-dissipation balance that are largely consistent with simple scaling relationships for the vertical divergence in TKE flux. Both the impact of stratification and deviation from equilibrium turbulence observed in the data are largely consistent with commonly used turbulence closure models that employ "nonequilibrium" stability functions. The data compare most favorably with the nonequilibrium version of the L. H. Kantha and C. A. Clayson stability functions. Not only is this approach more consistent with the observed critical gradient Richardson number of 0.25 , but it also accounts for the large deviations from equilibrium turbulence in a manner consistent with the observations.
\end{abstract}

\section{Introduction}

\section{a. Motivation}

Understanding turbulent mixing in the presence of strong density stratification is an important and unresolved problem in estuarine research. The ability to parameterize turbulent fluxes in the presence of strong stratification is important to understanding a wide array of both physical and biogeochemical processes. Given the difficulties in directly measuring turbulent fluxes, researchers often rely upon numerical circulation models to gain insight into fundamental estuarine processes (e.g., Li et al. 2006; Guo and Valle-Levinson 2008; Scully et al. 2009; Scully

Corresponding author address: Malcolm E. Scully, Center for Coastal Physical Oceanography, 4111 Monarch Way, 3rd Floor, Norfolk, VA 23508.

E-mail: mscully@odu.du
2010). Circulation models in estuarine environments typically parameterize turbulent processes using secondmoment turbulence closure models (Warner et al. 2005). The closure assumptions employed by these models often are based and validated against laboratory measurements, large eddy simulations, and direct numerical simulations (Kantha and Clayson 1994, hereafter KC94; Canuto et al. 2001, hereafter CA01). Laboratory and numerical studies of turbulence are limited to Reynolds numbers many orders of magnitude below those typically observed in energetic estuarine environments. Testing the applicability of these models to turbulence at geophysically relevant scales requires field measurements of both turbulent and mean flow quantities.

Because field measurements of turbulent quantities are extremely challenging to obtain, studies that directly test second-moment turbulence models using field data are rare. The few studies that compare field measurements of 
turbulent quantities with model simulations are generally inconclusive. Stacey et al. (1999) found that the 2.5-level model of Mellor and Yamada (1982) significantly underestimated the levels of turbulent kinetic energy (TKE) in the pycnocline region of a stratified estuary. Their results suggested that the model did not accurately account for the vertical divergence in the turbulent transport of turbulent kinetic energy. Similar results were found for a stratified region of the Irish Sea, and only when an empirical parameterization of internal waves was added did the model satisfactorily predict the observations (Burchard et al. 1998). However, Simpson et al. (2002) found good agreement between observed tidal variations in the turbulent dissipation rate and simulations employing a 1D model with the $k-\varepsilon$ model in another study in the Irish Sea. In the stratified Hudson River estuary, Peters and Baumert (2007) used a simplified closure model that assumes constant stability functions and found reasonable agreement between modeled and observed dissipation rates and turbulent length scales in regions of energetic mixing, but their model significantly underpredicted these quantities in strongly stratified areas near the pycnocline.

Despite these potential shortcomings, 3D modeling studies that employ these closure assumptions generally can hindcast mean hydrographic quantities in estuarine environments with good skill (Warner et al. 2005; Li et al. 2005; Ralston et al. 2010a). These studies show that the model's ability to reproduce mean quantities is largely insensitive to the choice of turbulence closure. From these results, it is tempting to conclude that the second-moment turbulence models essentially capture the necessary processes to sufficiently model stratified estuarine turbulence. However, it should be noted that, in most of these studies, the models are adjusted with the goal of reproducing observations and as such are not unbiased tests of the underlying parameterizations of turbulence. The few field studies that directly evaluate closure models generally only compare observations of one turbulent quantity (typically turbulent dissipation rate) using a single closure model. More detailed comparisons of the different formulations for the stability functions using field data are less common. This paper provides one such test of the performance of closure models that quantifies several turbulence quantities across a range of forcing conditions.

\section{b. Background}

Second-moment turbulence models rely on the downgradient assumption for the vertical transport of momentum $\left\langle u^{\prime} w^{\prime}\right\rangle$ and buoyancy B (Rodi 1980; Hossain 1980),

$$
-\left\langle u^{\prime} w^{\prime}\right\rangle=A_{z} \frac{\partial u}{\partial z} \quad \text { and } \quad B=\frac{g}{\rho_{o}}\left\langle\rho^{\prime} w^{\prime}\right\rangle=K_{z} N^{2}
$$

where the eddy viscosity $A_{z}$ and eddy diffusivity $K_{z}$ can be represented as the product of the relevant velocity and length scale of the turbulent motion. Following the notation of the $k-\varepsilon$ model, the turbulent length scale can be represented as

$$
L=\left(c_{\mu}^{o}\right)^{3} \frac{k_{q}^{3 / 2}}{\varepsilon},
$$

where $k_{q}$ is the TKE, which is defined as

$$
k_{q}=\frac{1}{2}\left(\left\langle u^{\prime 2}\right\rangle+\left\langle v^{\prime 2}\right\rangle+\left\langle w^{\prime 2}\right\rangle\right)
$$

$\varepsilon$ is the rate of dissipation of TKE; and $c_{\mu}^{o}$ is a constant defined as the ratio of stress to TKE for a constant-stress logarithmic boundary layer (Umlauf and Burchard 2003),

$$
\left(c_{\mu}^{o}\right)^{2}=\frac{-\left\langle u^{\prime} w^{\prime}\right\rangle}{k_{q}} .
$$

For unstratified flows near boundaries, where turbulent production and dissipation balance, these models are tuned so that the master turbulent length scale is consistent with boundary layer scaling $L_{\mathrm{BL}}$,

$$
L_{\mathrm{BL}}=\kappa z \sqrt{1-z / h_{\mathrm{BL}}},
$$

where $\kappa$ is the von Kármán constant $(\sim 0.41)$; $z$ is the vertical coordinate; and $h_{\mathrm{BL}}$ is the boundary layer height, where the stress approaches zero. This assumes that the local turbulent velocity scale is proportional to the square root of local value of TKE, which can be related to the stress via Eq. (4). Under stratified conditions, most models limit the master length scale so it does not exceed the Ozmidov scale $L_{O}$,

$$
L_{O}=\frac{\varepsilon^{1 / 2}}{N^{3 / 2}}
$$

The Ozmidov scale is observed to be the upper limit for the vertical length scale of turbulence in a stratified flow (Dillon 1982) and is the point at which buoyancy and inertial forces are equal. It should be noted that the limit of the Ozmidov scale is not an intrinsic property of most models but rather a numerical limit that is typically imposed on the length scale (i.e., Galperin et al. 1988).

Using the master length scale defined in (2) and assuming the relevant scale for turbulent velocity fluctuations is proportional to $k_{q}^{1 / 2}$, the eddy viscosity and eddy diffusivity can be represented as

$$
A_{z}=c_{\mu} \frac{k_{q}^{2}}{\varepsilon} \quad \text { and } \quad K_{z}=c_{\mu}^{\prime} \frac{k_{q}^{2}}{\varepsilon}
$$


where $c_{\mu}$ and $c_{\mu}^{\prime}$ are the nondimensional stability functions. Although some implementations assume constant values for the stability functions (Baumert and Peters 2004), other approaches assume that they are functions of the nondimensional stratification $\alpha_{N}$ and the nondimensional shear $\alpha_{S}$, given as

$$
\alpha_{N}=N^{2} \frac{k_{q}^{2}}{\varepsilon^{2}} \quad \text { and } \quad \alpha_{S}=\left(\frac{\partial u}{\partial z}\right)^{2} \frac{k_{q}^{2}}{\varepsilon^{2}} .
$$

Although much attention has focused on the difference between second-moment turbulence models (Mellor and Yamada 1982; $\mathrm{k}-\varepsilon$ and $\mathrm{k}-\omega)$, Umlauf and Burchard (2003) demonstrate that these models are structurally similar and consistent with the parameterization in (7). As a result, model performance depends more strongly on the choice of stability functions than on which two-equation model is used (e.g., Burchard et al. 1998).

The formulation of the stability parameters has significant consequences for how turbulence is modeled. In the simplest terms, the stability functions determine the ratio between the turbulent momentum flux and TKE. This quantity, which is sometimes referred to as the nondimensional stress, can be represented in terms of the stability function for momentum, using (1), (7), and (8) as

$$
\frac{-\left\langle u^{\prime} w^{\prime}\right\rangle}{k_{q}}=\sqrt{c_{\mu} \frac{P}{\varepsilon}}=c_{\mu} \sqrt{\alpha_{S}},
$$

where $P$ is shear production $\left(P=-\left\langle u^{\prime} w^{\prime}\right\rangle \partial u / \partial z\right)$. This ratio is a function of both the correlation coefficient between the horizontal and vertical velocity fluctuations (i.e., $r=-\left\langle u^{\prime} w^{\prime}\right\rangle /\left[\left\langle u^{\prime 2}\right\rangle\left\langle w^{\prime 2}\right\rangle\right]$ ) and the large-scale anisotropy, which is defined as

$$
A=\frac{2 k_{q}}{\left\langle w^{\prime 2}\right\rangle} .
$$

Using observations collected from the atmospheric boundary layer, Mauritsen and Svensson (2007) show that the large-scale anisotropy increases and the correlation coefficient decreases when the gradient Richardson number $\left(\mathrm{Ri}=N^{2}(\partial u / \partial z)^{-2}\right)$ exceeds a value of roughly 0.25 . Although some models try to represent the collapse of turbulence into a highly anisotropic and uncorrelated interval wave field in a physically realistic way (Baumert and Peters 2004), most simply constrain the turbulent flux to approach zero once some critical value of $\mathrm{Ri}$ $\left(\mathrm{Ri}_{\mathrm{cr}}\right)$ is exceeded. The choice of stability functions, not the choice of two-equation model, sets the value for
$\mathrm{Ri}_{\mathrm{cr}}$. The appropriate value for $\mathrm{Ri}_{\mathrm{cr}}$ is a matter of some debate. Miles (1961) and Howard (1961) theoretically show that $\mathrm{Ri}_{\mathrm{cr}}=0.25$ using linear stability theory. However, Abarbanel et al. (1984) include nonlinear interactions and derive a theoretical value of $\mathrm{Ri}_{\mathrm{cr}}=1$. The commonly used stability functions of $\mathrm{KC} 94$ predict a value of 0.235 , whereas CA01 predict $\mathrm{Ri}_{\text {cr }}=0.847$.

In addition to empirically parameterizing the influence of stratification on mixing through the correlation coefficient and/or large-scale anisotropy, the stability functions also modify the nondimensional stress to account for deviations from the first-order local TKE balance. For a unidirectional shear flow with the boundary layer approximation, the evolution equation for TKE can be written as

$$
\begin{aligned}
\frac{\partial k_{q}}{\partial t}+\mathbf{U} \cdot \nabla k_{q}= & -\overline{u^{\prime} w^{\prime}} \frac{\partial \bar{u}}{\partial z}+\frac{g}{\rho_{o}} \overline{\rho^{\prime} w^{\prime} x}-\frac{\partial}{\partial z}\left(\frac{1}{\rho_{o}} \overline{w^{\prime} p^{\prime}}\right) \\
& -\frac{\partial}{\partial z}\left(\overline{w^{\prime} k_{q}^{\prime}}\right)-\varepsilon .
\end{aligned}
$$

Here, the time evolution term and advective terms are on the left side of the equation. On the right-hand side, the first term is the turbulent shear production $P$, the second term is the buoyancy flux $B$, the third term is the pressure-gradient work, the fourth term is the divergence in vertical turbulent flux, and the last term is the turbulent dissipation $\varepsilon$. In many situations, a local TKE balance (i.e., $P+B=\varepsilon$ ) is assumed. Using the notation of turbulence closure, deviations from this so-called equilibrium condition can be presented as

$$
\alpha_{S} c_{\mu}-\alpha_{N} c_{\mu}^{\prime}=\frac{P+B}{\varepsilon} .
$$

The equilibrium condition is assumed to hold in the derivation of the "quasi equilibrium" stability functions. This approach is referred to as quasi equilibrium because only the derivation of the stability functions relies on the assumption of equilibrium conditions and the models retain the fully dynamic TKE equation (KC94). Based on a scaling analysis, Galperin et al. (1988) concluded that this is not a model inconsistency. A consequence of the equilibrium assumption is that the quasi-equilibrium models must use the downgradient assumption to quantify the divergence in vertical turbulent flux, where the eddy coefficient for the vertical transport of TKE is typically equated to the eddy viscosity of momentum. In contrast, the nonequilibrium model of CA01 does not rely on the assumption of equilibrium in deriving the stability functions, and the downgradient assumption for the vertical transport of TKE is not necessary. 


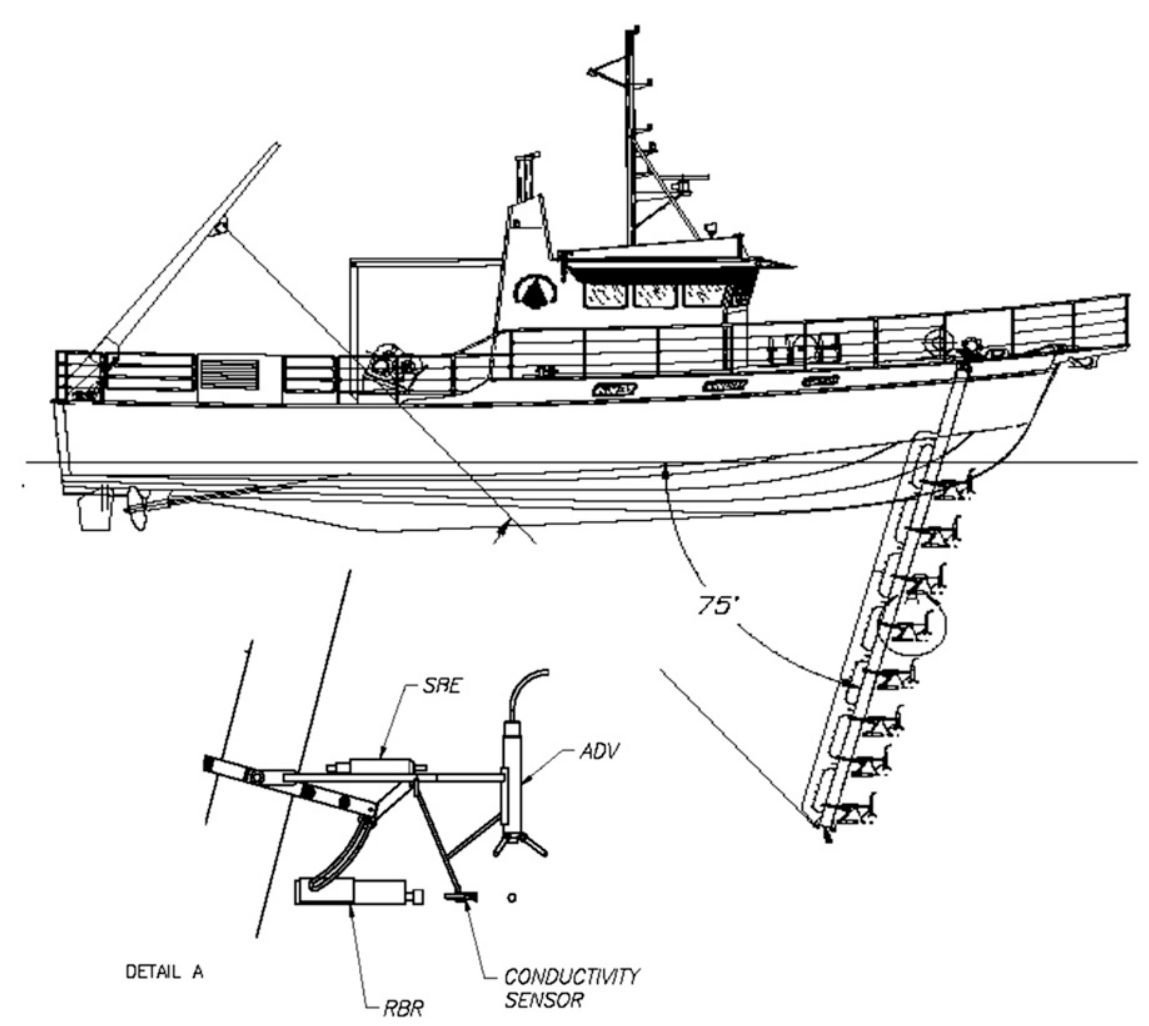

FIG. 1. Schematic of the MAST with detail of instrument bracket. Each bracket contains an ADV, SBE-07 microconductivity sensor, and RBR CTD. Only six instruments were used for this study.

\section{c. Objectives}

In this paper, we present measurements obtained by a new platform for measuring stratified turbulence in the coastal ocean that was designed to provide robust estimates of both turbulent and mean flow quantities. The goal is to use field observation to evaluate commonly used second-moment turbulence models, including several parameterizations for the stability functions. Direct field observations of turbulence will be used 1) to evaluate the consistency of observed turbulent spectra and cospectra with previously proposed universal forms; 2) to compare estimates of turbulent length scale with the theoretical limitation imposed by either boundary layer or Ozmidov scaling; 3 ) to examine the relationship between Ri and the large-scale anisotropy; and 4) to evaluate the dominant terms in the TKE equation, including the downgradient assumption for the vertical flux of TKE. These results will then be used to examine how well several commonly used stability functions account for both the influence of stratification and deviations from equilibrium turbulence. The stability functions considered will include 1) constant stability functions; 2) the quasi-equilibrium stability functions proposed by $\mathrm{KC} 94 ; 3)$ the nonequilibrium form of $\mathrm{CA} 01$; and
4) the nonequilibrium form of $\mathrm{KC} 94$ as derived in Burchard and Bolding (2001, hereafter BB01).

\section{Methods}

\section{a. MAST}

Data presented in this manuscript were collected using the Mobile Array for Sensing Turbulence (MAST; Geyer et al. 2008). The MAST is a 10-m rigid instrument package that is deployed vertically from a cross bar that mounts across the bow of a research vessel (Fig. 1). The MAST attaches to the cross bar via a universal joint, and a lift line attached to the end of the MAST is used to raise and lower the system, adjusting the angle depending on boat speed and water depth. For the measurements described here, six instrument brackets were located at evenly spaced intervals along the MAST. Each instrument bracket is adjustable so that the instruments can be maintained a constant angle relative to the water surface, regardless of the angle of the MAST. Each instrument bracket contains three collocated sensors: 1 ) a Sontek acoustic Doppler velocimeter (ADV; $25 \mathrm{~Hz}) ; 2$ ) a Seabird Electronics SBE-7 microconductivity probe $(300 \mathrm{~Hz})$; and 3) an RBR conductivity-temperature-depth (CTD) sensor 


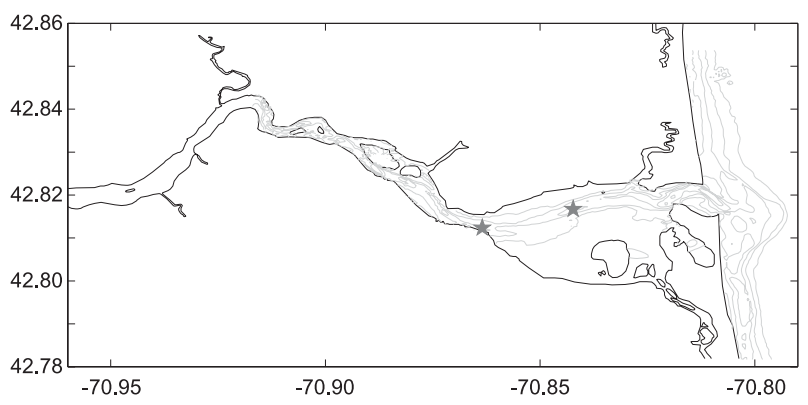

FIG. 2. Site map and bathymetry of Merrimack River field study. Stars denote ebb tide anchor station locations and the bathymetric contour interval is $2 \mathrm{~m}$. The western site was occupied on 12 and 13 May 2007, and the eastern site was occupied on 14 May 2007.

(6 Hz). The sampling of the instruments is synchronized in time allowing for direct turbulent fluxes to be measured. A Benthos altimeter is mounted on the bottom instrument bracket to measure the distance to the bed. A six-axis inertial motion package is affixed to the top of the MAST to measure the movement of the MAST.

\section{b. Study site}

The observations presented here were obtained during a deployment of the MAST in the Merrimack River estuary in May 2007. The Merrimack River is a highly stratified salt wedge estuary that enters the Atlantic Ocean near the border between Massachusetts and New Hampshire (Fig. 2). The spring tidal range is $2.75 \mathrm{~m}$ with tidal velocities approaching $1.5 \mathrm{~m} \mathrm{~s}^{-1}$. The annual-mean river discharge is approximately $250 \mathrm{~m}^{3} \mathrm{~s}^{-1}$ but typically exceeds $1500 \mathrm{~m}^{3} \mathrm{~s}^{-1}$ during high flow conditions. The mean discharge over the duration of the experiment was roughly $350 \mathrm{~m}^{3} \mathrm{~s}^{-1}$. During this experiment, measurements were made utilizing the MAST at fixed anchor stations as well as underway. In this paper, only the results collected during fixed anchor stations will be discussed. Two anchor stations in the region down river from where the estuary expands laterally were occupied during this experiment (Fig. 2). The first and westernmost location was occupied on 12 and 13 May 2007 and was located in the ebb-dominant channel. The second and more eastern location was occupied on 14 May 2007 and was located in the flood-dominant channel. At both locations, the anchor stations spanned the duration of the ebb tide.

\section{c. Analysis}

The data from this experiment were partitioned into 5-min bursts. The ADV data for each burst were rotated so that both the mean vertical velocity at each sensor level and the depth-averaged lateral velocity across the array were zero. The top ADV sensor malfunctioned in a manner that it could only be used for mean velocity but not for the turbulent quantities presented below. Data from the 5-min bursts were used to compute the mean flow statistics, including the mean horizontal velocity, salinity, and temperature. These data provide continuous and collocated estimates of the vertical shear and stratification giving highly resolved estimates of Ri.

Estimates of turbulent dissipation rate, velocity variance, and turbulent length scale were estimated from the ADV data as described below. Ship-based field measurements of these quantities are complicated by several factors. First, surface gravity waves produce nonturbulent wave orbital motion and induce boat motion, which moves the sensors through the water. As a result, when the amplitude of surface gravity waves is significant, there are nonturbulent motions that contaminate the velocity spectrum. Although it is theoretically possible (neglecting instrument noise) to remove all of the velocity that is induced by the movement of the sensors using the data from the inertial motion package affixed to the MAST, this does not address the nonturbulent wave motion. Further, the complex interaction between the boat-induced motions and the vertical structure of the wave velocities makes separating the two very challenging. The second complicating factor is the noise limitations of the ADV sensors. Under strongly stratified and/or low turbulent energy conditions, significant portions of the velocity spectra may fall below the noise floor. As a result, under low energy conditions the $-5 / 3$ slope region of the inertial subrange may be obscured by noise, precluding accurate use of the inertial dissipation method (e.g., Grant et al. 1984).

To avoid the complications introduced by both nonturbulent wave and boat motions and limitations imposed by the ADV noise floor, the data are interpreted using the spectral model proposed by Kaimal et al. (1972). Their results demonstrate that the one-sided wavenumber $k$ autospectra of turbulent velocity fluctuations collected in the atmospheric boundary layer exhibit a universal form when properly normalized, which is given as

$$
\frac{k S_{\beta \beta}(k)}{\left\langle\beta^{\prime 2}\right\rangle}=\frac{0.16\left(k / k_{o}\right)}{1+0.16\left(k / k_{o}\right)^{5 / 3}},
$$

where $\beta$ indicates either the vertical ( $w)$ or horizontal $(u$ and $v$ ) turbulent velocity component; $\left\langle\beta^{\prime 2}\right\rangle$ is the turbulent velocity variance in that direction; and $k_{o}$ is the wavenumber associated with the dominant energy containing scales. At high wavenumber, the dimensional form of (13) asymptotes to

$$
S_{\beta \beta}(k)=\left\langle\beta^{\prime 2}\right\rangle k_{o}^{2 / 3} k^{-5 / 3} .
$$


This is consistent with Kolmogorov's inertial subrange model, because Kaimal et al. (1972) define

$$
k_{o}=\alpha^{3 / 2} \frac{\varepsilon}{\left\langle\beta^{\prime 2}\right\rangle^{3 / 2}}
$$

where $\alpha$ is the Kolmogorov constant, which we assume is equal to 0.51 for fluctuations parallel to the direction of the mean flow ( $\mathrm{u}$ in our notation) and $4 / 3$ times $0.51=$ 0.68 for fluctuations perpendicular to the direction of mean flow ( $\mathrm{v}$ and $\mathrm{w}$ in our notation), which is consistent with an isotropic inertial-range model (e.g., Tennekes and Lumley 1972). Assuming that the peak of the variancepreserving spectrum $\left(k=3.8 k_{o}\right)$ given by (13) occurs at the dominant length scale of turbulent motion $L_{\beta}$ gives

$$
L_{\beta}=\frac{1}{3.8 k_{o}}=\frac{1}{3.8 \alpha^{3 / 2}} \frac{\left\langle\beta^{\prime 2}\right\rangle^{3 / 2}}{\varepsilon} .
$$

Fitting this model to observed spectrum gives estimates of both the velocity variance and turbulence length scale, which in turn provides an estimate of the dissipation rate [via Eq. (16)].

To fit the observed spectra with the proposed model of Kaimal et al. (1972), we must omit contributions from waves, sensor motion, and instrument noise. Because of the geometry of the ADV sensor, the noise variance in the direction perpendicular to the sensor orientation is roughly a factor of 30 lower than in the direction parallel to sensor orientation (Voulgaris and Trowbridge 1998). Therefore, the initial efforts to estimate the velocity variance, turbulent length scale, and dissipation rate use the vertical velocity spectra. Consistent with previous studies, the model proposed by Kaimal et al. (1972) is fit to observed vertical velocity spectrum using a twoparameter least squares minimization (e.g., Gerbi et al. 2008). Before fitting the spectra, all spectral energy found in the frequencies where surface wave energy was observed $(0.15>$ freq $>0.85)$ and all spectral energy below the estimated noise floor $\left(8 \times 10^{-2} \mathrm{~cm}^{2} \mathrm{~s}^{-2}\right)$ based on the results of Voulgaris and Trowbridge (1998) were excluded. Inside the sheltered and fetch-limited Merrimack River, significant wave and boat-induced motions were limited to this relatively narrow frequency band. The observed noise floor of the ADVs used in this experiment was generally below that reported by Voulgaris and Trowbridge (1998), but this value is used as a conservative estimate to ensure that spectral fits are not contaminated by noise in the high-wavenumber portion of the spectrum. Spectra are then converted from the frequency domain to the wavenumber domain using the observed horizontal velocity and Taylor's frozen turbulence hypothesis. Use of the frozen turbulence hypothesis generally did not result in contamination of the high-wavenumber portion of the spectra by wave advection because the wave orbital velocities were generally an order of magnitude below mean current speeds (Lumley and Terray 1983). The vertical velocity spectra are then fit with the spectral model to provide estimates of vertical velocity variance, turbulent length scale $L_{w}$, and turbulent dissipation rate. The estimates of the dissipation rate obtained from the spectral fit are analogous to estimates obtained from the inertial dissipation method, but with the advantage that contributions due to instrument noise are effectively removed.

With the sensor configuration used in this experiment, a well-resolved inertial subrange is typically observed in the vertical velocity spectra, but much of the inertial subrange of the horizontal velocity spectrum is obscured by the higher noise in this component of velocity. With poor resolution of the high-wavenumber horizontal velocity spectra, the two-parameter fitting procedure described above could not be used to provide reliable estimates of horizontal velocity variance and integral wavenumber $k_{o}$. However, the observed horizontal velocity spectrum can still be fit with this model if the dissipation estimates obtained from the vertical velocity spectrum are used, and it is assumed that the isotropic Kolmogorov relation holds over the inertial subrange. This method was used successfully by Gerbi et al. (2009) to estimate of the horizontal velocity variances in an environment dominated by surface gravity waves. This assumes that the high-wavenumber portion of the horizontal velocity spectrum (the inertial subrange) is fixed based on the estimate of dissipation from the vertical velocity spectra and allows the low-wavenumber portion of the horizontal velocity spectra to be fit. Consistent with the methods used to fit the vertical velocity spectra, frequencies in the wave band and all portions of the spectra below the estimated horizontal velocity noise floor $\left(\sim 2.4 \mathrm{~cm}^{2} \mathrm{~s}^{-2}\right.$; following Voulgaris and Trowbridge 1998) are omitted. Again, this was a conservative estimate of the noise floor based on the data but ensures instrument noise does not adversely affect the spectral fits. By assuming that the inertial subrange of the horizontal spectra are fixed given the dissipation rate estimated from the vertical velocity spectra, there is only one free parameter left to fit: the horizontal velocity variance.

Estimates of both momentum flux and buoyancy flux were obtained using methods similar to those described above. This approach has been applied to estimate momentum flux by Trowbridge and Elgar (2003) and more recently by Gerbi et al. (2008). Both papers use the results of Kaimal et al. (1972), who suggest that nondimensional cospectra have the following form: 


$$
\frac{k S_{\beta \mathrm{w}}(k)}{\left\langle\beta^{\prime} w^{\prime}\right\rangle}=\frac{0.88\left(k / k_{o}\right)}{1+1.5\left(k / k_{o}\right)^{7 / 3}} .
$$

This is analogous to (13), but $S_{\beta \mathrm{w}}$ represents the cospectral energy of the vertical velocity fluctuations and either the horizontal velocity or density fluctuations. Consistent with (13), cospectrum are normalized by the covariance and integral wavenumber $k_{o}$. Again, all energy in the wave band frequencies is omitted prior to transforming the data into wavenumber spectra using the Taylor's frozen turbulence hypothesis. We assume any covariance at the highest resolved wavenumbers is correlated noise. This is removed by subtracting the mean covariance at frequencies greater than $5 \mathrm{~Hz}$ from the observed cospectra prior to transforming the data into wavenumber. Turbulent fluxes are then obtained by fitting the observed cospectra to the model using a twoparameter least squares optimization. Both Trowbridge and Elgar (2003) and Gerbi et al. (2008) used similar methodology in environments with high wave energy and demonstrated reliable estimates of turbulent fluxes and length scales. We extend their approach to include estimates of buoyancy flux as well, using the collocated ADV and SBE-7 sensors, calibrated for density (which is dominated by salinity in this highly stratified salt wedge estuary). The temperature and salinity data measured by the RBR were used to calibrate the SBE-7 data to give high-frequency estimates of turbulent density variations. In estimating both momentum flux and buoyancy flow, some cospectra deviated considerably from the model and could not be fit with physically reasonable parameters. These data were excluded from further analysis.

There were numerous times during the experiment when surface waves were absent. To assess the spectral fitting procedure, both the autospectra and cospectra were fit to these data with the models presented above, omitting energy in the frequency bands where waves were observed during other time periods. These spectral estimates obtained when surface waves were absent were then compared with direct integrals of the variance and covariance as a check on the methodology. The difference between the spectral fits and the direct integrals was less than $5 \%$ for all components of velocity variance as well as momentum and buoyancy flux estimates (data not shown).

\section{Results}

At both sites, the toe of the salt wedge is located upestuary from the anchor station location at the beginning of the ebb tide. As the tide turns, the near-surface velocities increase rapidly while the lower layer remains

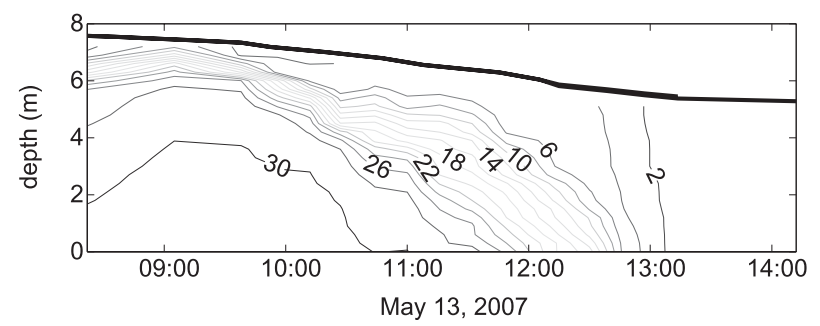

FIG. 3. Time evolution of salinity field spanning the ebb tide, observed at the western anchor station on 13 May 2007. Contour interval is 2 psu.

arrested, leading to strong mixing in a middle water column free shear layer. Eventually, the shear layer couples into the bottom boundary layer and the toe of the salt wedge is advected seaward. By the end of the ebb tide, the entire salt field has been advected seaward of the anchor station location and unstratified boundary layer mixing is observed. A representative time series of the salinity field is presented in Fig. 3. This general pattern was observed during all three of the ebb tide anchor stations (for more details, see Ralston et al. 2010b).

\section{a. Normalized velocity spectra}

Many of the turbulent quantities presented in this paper rely on fitting a theoretical spectral form to observed velocity spectra. To test the consistency of the data with the proposed theoretical spectra, the observed velocity autospectra have been nondimensionalized following Eq. (13) (Fig. 4). For each plot, all of the individual spectra are plotted, as are the spectral values obtained from averaging all spectral data in logarithmically spaced intervals of $k / k_{o}$. In all cases, data within the wave band have been omitted, as have all values below the estimated noise floors. For all three components of velocity, when $\mathrm{Ri}<0.25$ there is good agreement between the observed velocity spectra and the form proposed by Kaimal et al. (1972). In all three velocity components, both the peak of the variance-preserving spectra and the low-wavenumber portion of the spectrum are generally consistent with the Kaimal model. Because of the lower noise floor, a well-resolved inertial subrange (with a slope $\sim k^{-2 / 3}$ in the normalized form) is evident in the vertical velocity spectra. Although the higher noise floor in the horizontal components of velocity prevents resolution of a large portion of the inertial subrange, the roll off and transition to the inertial subrange are consistent with the model.

Under conditions when $\mathrm{Ri}>0.25$, the spectra are less consistent with the proposed model. The vertical velocity spectra agree reasonably well for values of $k / k_{o}>1$, but there is deviation at low wavenumber. In the vertical velocity spectra, the elevated low-wavenumber energy 

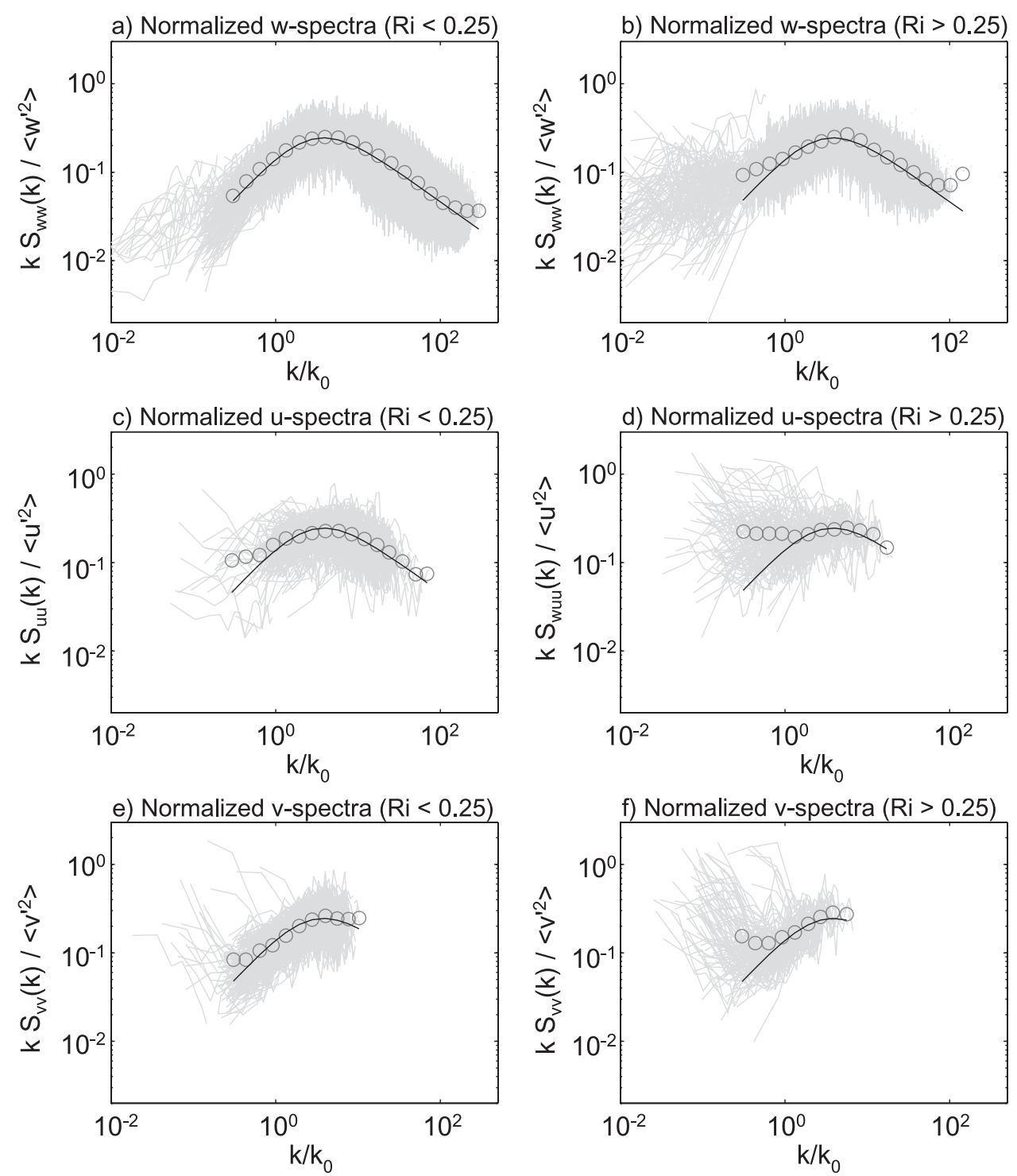

FIG. 4. Normalized velocity autospectra, segregated by gradient Richardson number Ri. Each individual spectrum is plotted (light gray lines), as are bin-averaged data (circles). Sold black line is the proposed nondimensional spectra of Kaimal et al. (1972). Spectra shown are for (a) vertical velocity for $\mathrm{Ri}<0.25$; (b) vertical velocity for $\mathrm{Ri}>0.25$; (c) horizontal velocity ( $u$ component) for $\mathrm{Ri}<0.25$; (d) horizontal velocity ( $u$ component) for $\mathrm{Ri}>0.25$; (e) horizontal velocity ( $v$ component) for $\mathrm{Ri}<0.25$; and (f) horizontal velocity ( $v$ component) for $\mathrm{Ri}>0.25$.

is spectrally separated from the peak of the variancepreserving spectra. However, this spectral gap is not evident in the horizontal velocity spectra when $\mathrm{Ri}>0.25$. This is particularly evident in the u component of velocity, where the peak of the variance-preserving spectrum is no longer distinguishable from the lower wavenumber energy. There are several possible reasons for these deviations. First, spectral models of turbulence generally assume stationarity. The applicability to actively growing shear instabilities is not established. During the initial roll up of a shear instability, there may be inputs of energy at low wavenumber before it is transferred to smaller scales during the collapse of secondary instabilities or billows (Smyth et al. 2001). Such growing instabilities are unlikely to have spectral characteristics consistent with the Kaimal et al. (1972) model and may exhibit elevated energies at lower wavenumbers. Alternatively, when the theoretical critical value of $\mathrm{Ri}$ is exceeded, turbulent motions may collapse into nonturbulent wave motions. Without the turbulence to transfer energy to higher wavenumber, it is unlikely that the spectral model would hold for these conditions. 

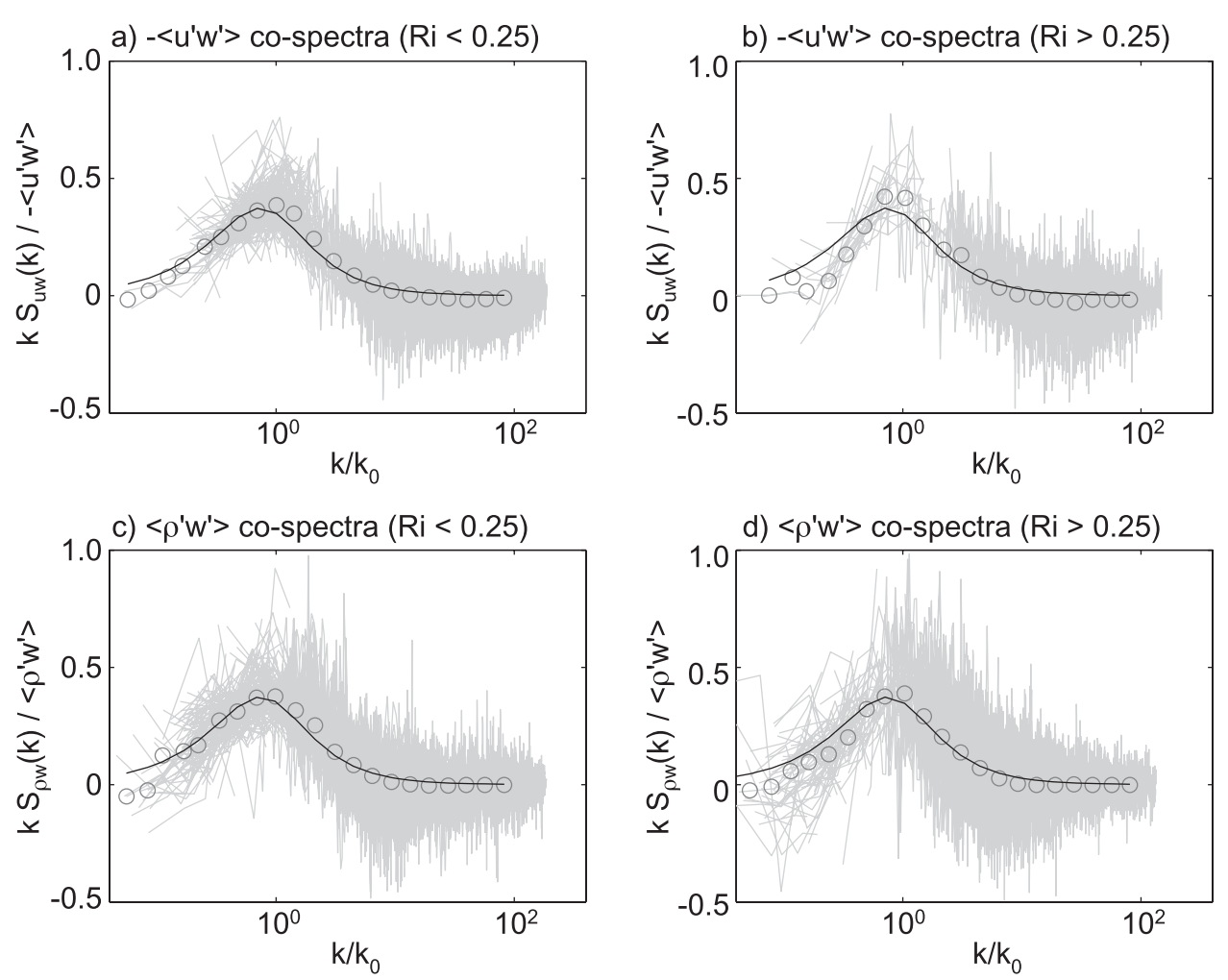

FIG. 5. Normalized momentum and buoyancy flux cospectra, segregated by gradient Richardson number Ri. Each individual cospectrum is plotted (light gray lines), as are bin-averaged data (circles). Sold black line is the proposed nondimensional cospectra of Kaimal et al. (1972). Spectra shown are for (a) momentum where $\mathrm{Ri}<0.25$; (b) momentum where $\mathrm{Ri}>0.25$; (c) buoyancy where $\mathrm{Ri}<0.25$; and (d) buoyancy where $\mathrm{Ri}>0.25$.

\section{b. Estimates of momentum and buoyancy flux}

Both the observed momentum and buoyancy cospectra agree well with the proposed model when properly normalized (Fig. 5). In all cases, the observed cospectra are slightly greater than the Kaimal model immediately to the right of the peak of the variance-preserving cospectrum and slightly less than the Kaimal model at high wavenumber. This indicates that the observed spectral slope is slightly greater than the $-7 / 3$ predicted by the model in the inertial subrange. Although this may represent a real difference, it is more likely a consequence of assuming that covariance at the highest resolved frequencies is correlated noise. Unlike the velocity spectra, there are no obvious differences between the observed cospectra based on the value of $\mathrm{Ri}$ (Fig. 5). However, it is important to point out that, for most of the data where $\mathrm{Ri}>0.25$, estimates of dissipation exceed the sum of shear production and buoyancy flux (see section 3e).

\section{c. Turbulent length scale}

Fitting the vertical velocity spectra with the model proposed by Kaimal et al. (1972) provides an estimate of the vertical velocity variance $\left\langle w^{\prime 2}\right\rangle$ and the integral wavenumber $k_{o}$. Thus, the Kaimal fits provide the data necessary to test the theoretical constraints on the turbulence length scale for different forcing conditions. Figure 6 compares the turbulent length scale calculated from the vertical velocity spectrum to the Ozmidov scale after normalizing each quantity by boundary layer scaling. The diagonal dashed line represents the asymptote of Ozmidov scaling (i.e., $L_{w}=L_{O}$ ) and horizontal dashed line represents the asymptote of boundary layer scaling (i.e., $L_{w}=L_{\mathrm{BL}}$ ). For the well-mixed conditions observed at the end of the ebb tide when $L_{O} \gg L_{\mathrm{BL}}$, the ratio $L_{W} /$ $L_{\mathrm{BL}}$ is roughly equal to one, consistent with the expected boundary layer scaling. In contrast, under strong stratification, there is a rapid decrease in the estimated turbulent length scale, which is generally equal to or less than the estimated Ozmidov scale. Only rarely does the turbulent length scale exceed the Ozmidov scale. In fact, less than $3 \%$ of the data have $L_{W}>L_{O}$ and nearly all these data are characterized by estimates of dissipation that significantly exceed production (see section 3e). In fact, on average, dissipation exceeds production by a factor of 2 for the outlying data. 


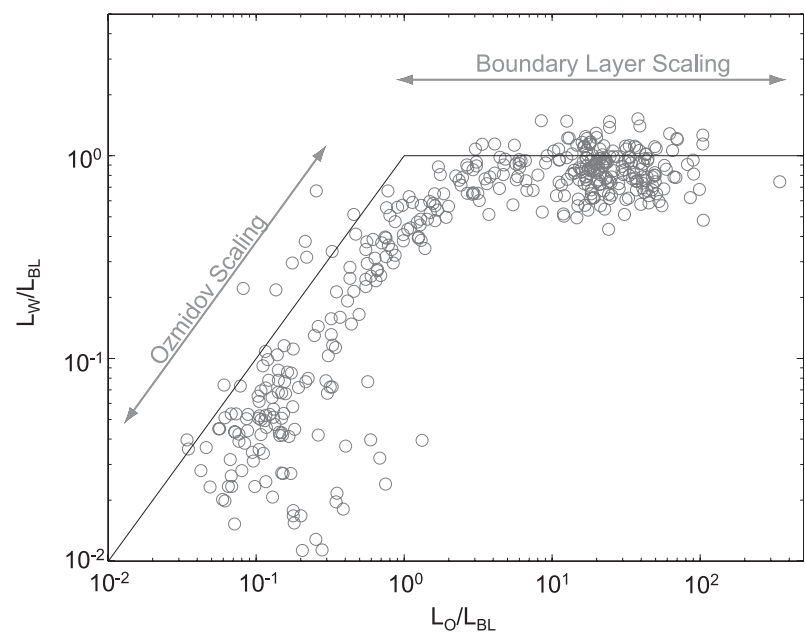

FIG. 6. Estimates of Ozmidov scale $L_{O}$ vs turbulent length scale estimated from vertical velocity spectra $L_{W}$ where each quantity has been normalized by boundary layer scaling $L_{\mathrm{BL}}$. The horizontal black line represents the limit of boundary layer scaling $\left(L_{W}=\right.$ $L_{\mathrm{BL}}$ ), and the diagonal black line represents the limit of Ozmidov scaling $\left(L_{W}=L_{O}\right)$

\section{d. Estimates of anisotropy}

With the spectral fitting techniques employed in this study, we are assuming isotropy at the dissipation scales of turbulence. Gargett et al. (1984) found that this was a reasonable assumption under energetic conditions when a well-resolved inertial subrange was observed. Although the methodology employed assumes isotropy at small scales, the large-scale anisotropy can still be estimated using the velocity variance obtained from the spectral fits. Figure 7a shows the estimated large-scale anisotropy [Eq. (10)] as a function of Ri. Under conditions when $\mathrm{Ri}$ is small, estimates of the anisotropy are generally consistent with expected values for unstratified boundary layer flows (e.g., $A=5.5$; Turner 1973). For conditions when $\mathrm{Ri}<0.1$, the mean value of $A$ is 5.4, with a slight trend for A to decrease with increasing Ri. However, as values of $\mathrm{Ri}$ increase there is a rapid increase in anisotropy, with a transition consistent with the MilesHoward linear stability threshold of $\mathrm{Ri}=0.25$.

Under boundary-limited conditions (i.e., $L_{O}>L_{\mathrm{BL}}$ ) the large-scale anisotropy varies only weakly as a function of $\alpha_{N}$ (Fig. 7b) and increases as a function of $\alpha_{S}$ (Fig. 7c). As the shear increases, more energy is fed into the horizontal component of velocity, increasing the anisotropy. Further, for boundary-limited conditions the shear is driven by the presence of the boundary, which limits the vertical scale of turbulent motion. As a result, the level of anisotropy increases with proximity to the bed, where shear is largest and the vertical length scale is increasingly constrained. The dependence of the anisotropy on the shear for boundary-limited conditions explains the slight negative relationship between $A$ and $\mathrm{Ri}$ seen for low values of $\mathrm{Ri}$.

In contrast, under Ozmidov-limited conditions (i.e., $L_{O}<L_{\mathrm{BL}}$ ), anisotropy is generally higher than 5.5, and the degree of anisotropy increases as a function of both increasing $\alpha_{N}$ (Fig. 7b) and increasing $\alpha_{S}$ (Fig. 7c). Because Ozmidov-limited conditions occur at higher values of Ri, the overall anisotropy for a given value of $\alpha_{N}$ or $\alpha_{S}$ is higher than for boundary-limited conditions. Without the influence of the boundary, the shear is more strongly related to the overall degree of stratification for Ozmidovlimited conditions. Increases in shear respond to increases in stratification, which increases the anisotropy by feeding more energy into the horizontal velocity component through increased shear production. The stratification limits the vertical scale of turbulent motion and removes energy from the vertical component of velocity through buoyancy production, enhancing the anisotropy. In
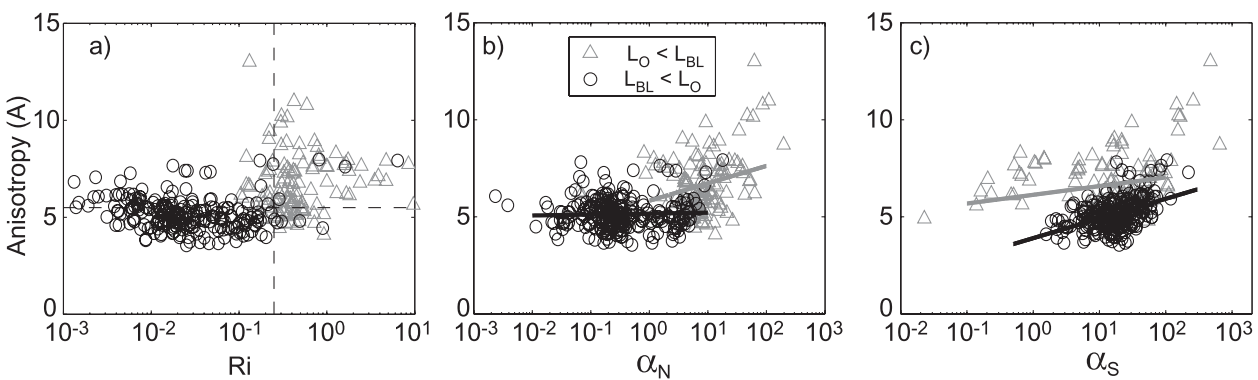

FIG. 7. Large-scale anisotropy $A$ plotted as a function of (a) gradient Richardson number Ri; (b) nondimensional stratification; and (c) nondimensional shear. Gray triangles are used to denote Ozmidovlimited conditions, and black circles represent boundary-limited conditions. In (a), the dashed vertical line indicates $\mathrm{Ri}=0.25$, which appears to represent the threshold beyond which there is a rapid increase in anisotropy. The dashed horizontal line indicates the suggested anisotropy for unstratified boundary layer conditions $(A=5.5)$ following Turner (1973). Black and gray lines in (b) and (c) represent regressions fit to the boundary-limited and Ozmidov-limited data, respectively. 
contrast to boundary-limited conditions, the overall anisotropy goes up with increasing Ri for Ozmidov-limited conditions.

One interpretation of the rapid increase in anisotropy for values of $\mathrm{Ri}>0.25$ is the increased contribution of nonturbulent wave motion. As noted above, the horizontal spectra begin to deviate from the Kaimal form in this range and the spectral gap between turbulent motion and wave energy disappears. This could lead to an overestimate of horizontal velocity variance and result in an upward bias of the anisotropy values at high Ri. However, the deviation in spectral shape and the abrupt transition in estimated anisotropy that occurs in the data when $\mathrm{Ri}$ exceeds 0.25 suggest the suppression of local turbulent production in this regime. As will be discussed below, although we do have estimates of momentum flux for conditions when $\mathrm{Ri}>0.25$, these generally occur when turbulent dissipation exceeds shear production, suggesting the suppression of local turbulent production.

\section{e. Turbulent kinetic energy balance}

The terms estimated from the spectral data allow examination of several terms in the turbulent kinetic energy balance, including the time rate of change, $P, B$, and $\varepsilon$. Under some conditions, direct estimates of the vertical TKE flux were possible using methods similar to those outlined in section 2c. However, because the vertical gradient in the flux is required, these estimates were generally very noisy and are not included in this analysis. Instead, two scaling relationships for the divergence in the vertical turbulent transport of TKE are presented for the two types of mixing observed during this experiment: 1) boundary layer mixing (appendix A) and 2) shear layer mixing (appendix B). These scaling relationships are used to provide an order of magnitude estimate of the importance of the turbulent transport term and a qualitative examination of how this term is expected to vary in the vertical. The advective terms and pressure-gradient work cannot be addressed with this dataset.

It is often assumed that the first-order TKE balance can be simply represented as $P+B=\varepsilon$. For both boundary-limited and Ozmidov-limited conditions, deviations from this first-order balance are observed (Fig. 8). The deviations are generally greater at the western sampling location (slope $=0.51 \pm 0.07$ ) as compared to the eastern site (slope $=0.63 \pm 0.06$ ), but the slopes of the regressions are not statistically different at the $95 \%$ confidence level. To investigate the potential that the observed imbalance in the TKE budget is due to the divergence in the turbulent transport during unstratified conditions, we use the boundary layer scaling presented in appendix A. This scaling is only applied to unstratified conditions, defined as data where the maximum observed

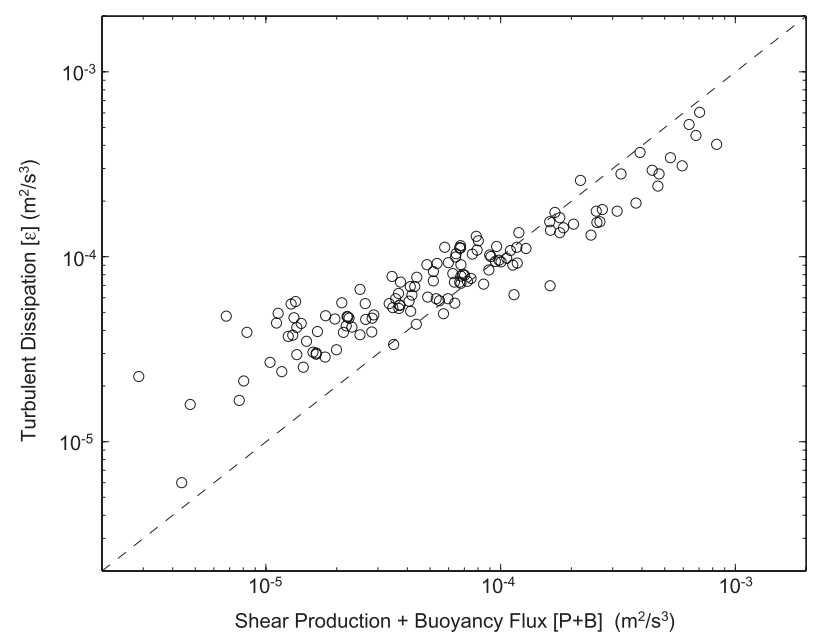

FIG. 8. Estimates of the local TKE balance where shear production $P$ plus buoyancy production $B$ are plotted against the dissipation rate $\varepsilon$. The dashed line represents local balance where $P+B=\varepsilon$.

water column value of $N^{2}<5 \times 10^{-4} \mathrm{~s}^{-2}$. For these conditions, the distance to the boundary sets the turbulent length scale, the velocity shear is logarithmic (Fig. 9a), and the profiles of stress decrease linearly away from the bed (Fig. 9b). The observed logarithmic shear and linear stress profiles give a vertical profile of the observed eddy viscosity that generally agrees with the theoretical parabolic form [Eq. (A2)]. Although the observed eddy viscosity is slightly smaller than the proposed scaling, the general vertical structure is consistent (Fig. 9c). The vertical profile of TKE decreases linearly away from the bed during unstratified conditions in a manner that is roughly linearly proportional to the observed stress profiles (Fig. 9d).

Figure 10a shows the vertical profiles of turbulent production and dissipation, averaged over the same data used in Fig. 9 (buoyancy flux is negligible under these conditions). For these data, turbulent production slightly exceeds dissipation near the bed, whereas dissipation increasingly exceeds production moving higher in the water column. This behavior is highlighted by dividing the average profile of measured shear production by the profile of measured dissipation (Fig. 10b). The nondimensional ratio based on the simple theory (A5) is shown for comparison. Although the observed magnitude of the ratio of production to dissipation is slightly less than predicted by the scaling, the vertical distribution is consistent with the simple analytic prediction suggesting that the divergence in turbulent transport is a plausible explanation for the observed imbalances seen in Fig. 8. This suggests that the elevated dissipation that is observed higher in the water column may be substantially balanced by the divergence in vertical TKE flux. Although we do not resolve the 

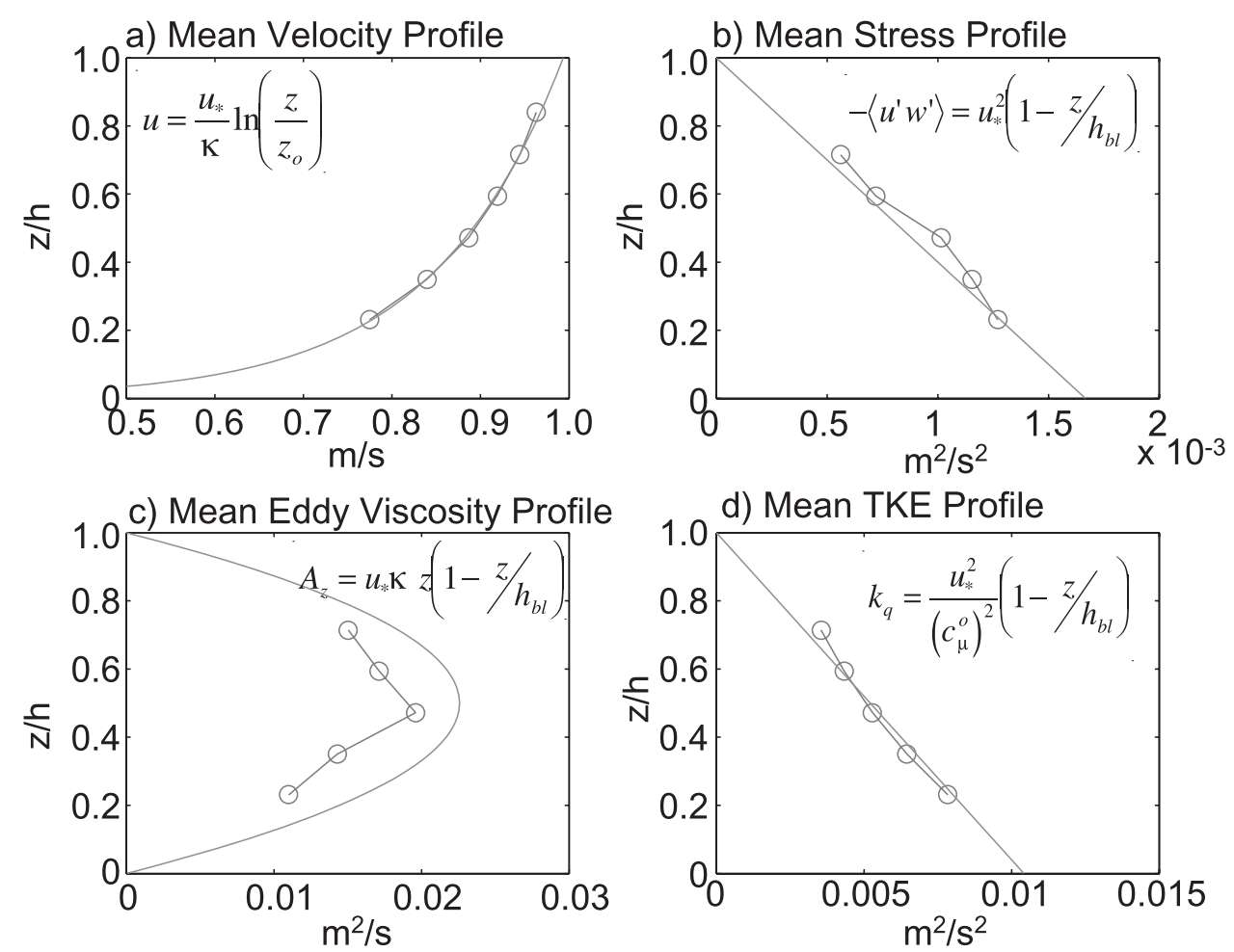

d) Mean TKE Profile

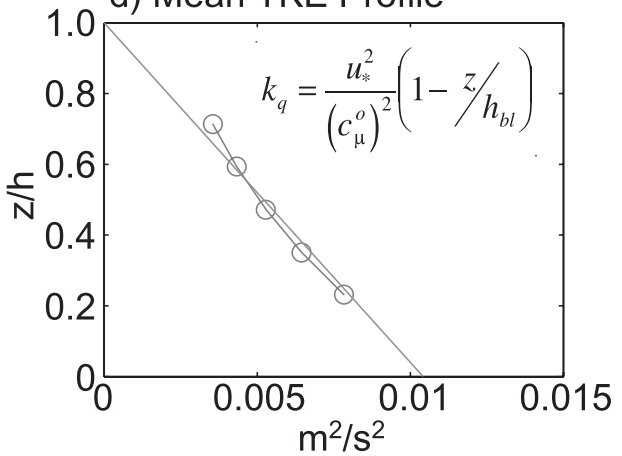

FIG. 9. Observed (circles) vertical profiles of (a) velocity; (b) stress; (c) eddy viscosity; and (d) TKE for unstratified conditions (end of ebb) compared to logarithmic boundary layer predictions (solid lines). Profiles are obtained by averaging all data where the minimum water column value of $N^{2}<5 \times 10^{-4} \mathrm{~s}^{-2}$.

lowest $1 \mathrm{~m}$ of the water column, there is evidence that production exceeds dissipation in this region and is a source of TKE to the upper half of the boundary layer. This scaling suggests that for unstratified conditions production goes to zero at the top of the boundary layer, where the dominant balance is between the divergence in vertical flux and dissipation.

a) Profiles $P$ and $\varepsilon$

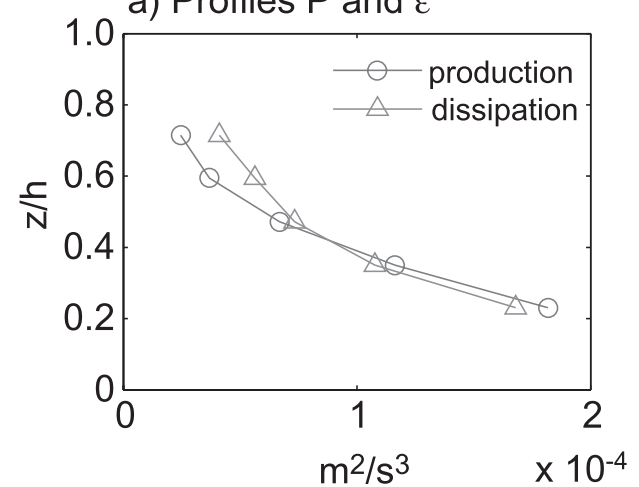

The nondimensional expression given in (A5) is only a function of the vertical location within the boundary layer and several reasonably well-constrained constants. Thus, for unstratified conditions, the observed dissipation rates can be "corrected" to account for the possible contribution from the divergence in the vertical flux of TKE by multiplying the observed dissipation by the

FIG. 10. (a) Vertical profiles of shear production $P$ and dissipation rate $\varepsilon$ averaged over all data where the minimum water column value of $N^{2}<5 \times 10^{-4} \mathrm{~s}^{-2}$. (b) Vertical profiles of the ratio of production to dissipation for the same data, compared to the prediction based on the boundary layer scaling that accounts for the vertical divergence in turbulent TKE transport [Eq. (A5)]. 

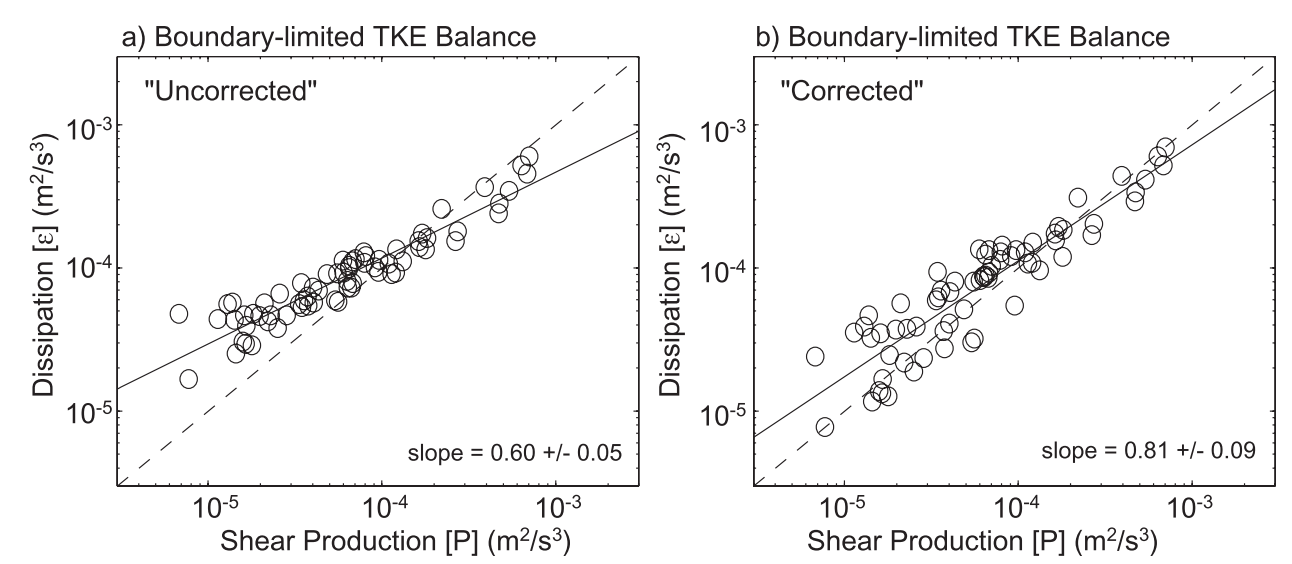

FIG. 11. Estimates of the local TKE balance for unstratified data. Shear production is compared to (a) the observed dissipation rate and (b) the corrected dissipation rate after applying a correction for the potential influence of the vertical divergence in turbulent TKE transport [Eq. (A5)]. The solid line is the best-fit least squares regression to the log-transformed data with the regression slope and $95 \%$ confidence interval. The dashed line represents $P=\varepsilon$.

expression given in (A5) assuming that the boundary layer height equals the local water depth. The observed shear production is compared to both the corrected and uncorrected dissipation in Fig. 11 for the unstratified conditions observed at the end of ebb. Despite this relatively simple approach, the slope of the regression is closer to unity when the influence of vertical TKE flux is accounted for via Eq. (A5), and the slopes of the regressions for the corrected and uncorrected data are statistically different at the $95 \%$ confidence interval (slope uncorrected $=0.60 \pm 0.05$; slope corrected $=0.81 \pm 0.09$ ). In applying this approach, it is assumed that the ratio of stress to TKE is constant. However, as will be discussed in section $3 \mathrm{f}$, this ratio is reduced when $\varepsilon>\mathrm{P}$ and would be expected to decrease in the vertical. Applying a linear correction for this effect $\left\{\right.$ i.e., $\left.\left(c_{\mu}^{o}\right)^{2}=0.2[1-(z / h)]\right\}$ results in a slope to the regression that is not statistically different from unity (slope $=0.98 \pm 0.13$ ) but increases the overall scatter.

A similar approach is used for the stratified shear layer mixing that is observed during the early and middle portions of the ebb tide, when the salt wedge is arrested and there is only weak velocity near the bed. Stress is generated by the strong shear across the pycnocline. As the ebb progresses and the salt wedge is advected seaward, the height of the interface descends until the stress from the shear layer couples into the bottom boundary layer. To account for the vertical changes in the location of the interface during the tidal cycle, a new vertical coordinate system is defined such that $z_{\mathrm{sl}}=0$ at the location in the water column where the magnitude of the observed shear is maximal. This is determined by differencing the velocity between the six instrument brackets and interpolating the shear back onto the original sensor locations assuming the velocity at the bed in zero and that the shear is constant between the top sensor and the water surface. The results are not sensitive to this interpolation, and the shear maximum is generally located between the second and fifth sensor locations.

Using this vertical coordinate system, all Ozmidovlimited data are averaged over evenly spaced bins based on the nondimensional distance from the center of the shear layer (i.e., $z_{\mathrm{sl}} / h_{\mathrm{sl}}=0$ ). The average velocity profile for these conditions is consistent with the hyperbolic tangent form used in Eq. (B2) in the appendix (Fig. 12a). Under these Ozmidov-limited conditions, the location of the stress maxima generally coincides with the center of the shear layer $\left(z_{\mathrm{sl}} / h_{\mathrm{sl}}=0\right)$ and decreases outward from this location (Fig. 12b). Although this stress distribution is generally consistent with the form assumed in appendix B, the observed stress distribution is asymmetric with slightly higher values of stress observed above the interface. There are also deviations at the lowermost location that may reflect the influence of the boundary. Near-bottom velocities slowly increase during the ebb until the shear layer couples into the bottom boundary layer and may enhance the stress near the bed before the salt wedge is fully advected seaward of the location.

Average profiles of buoyancy flux, shear production, and dissipation all show maximum values in the interface (Fig. 12c). In the interface, the sum of the shear production and buoyancy flux slightly exceeds dissipation. However, moving away from the center of the shear layer, the dissipation increasingly exceeds the TKE that is produced through shear production and lost to buoyancy flux. The overall vertical structure of the ratio $(P+B) / \varepsilon$ is 

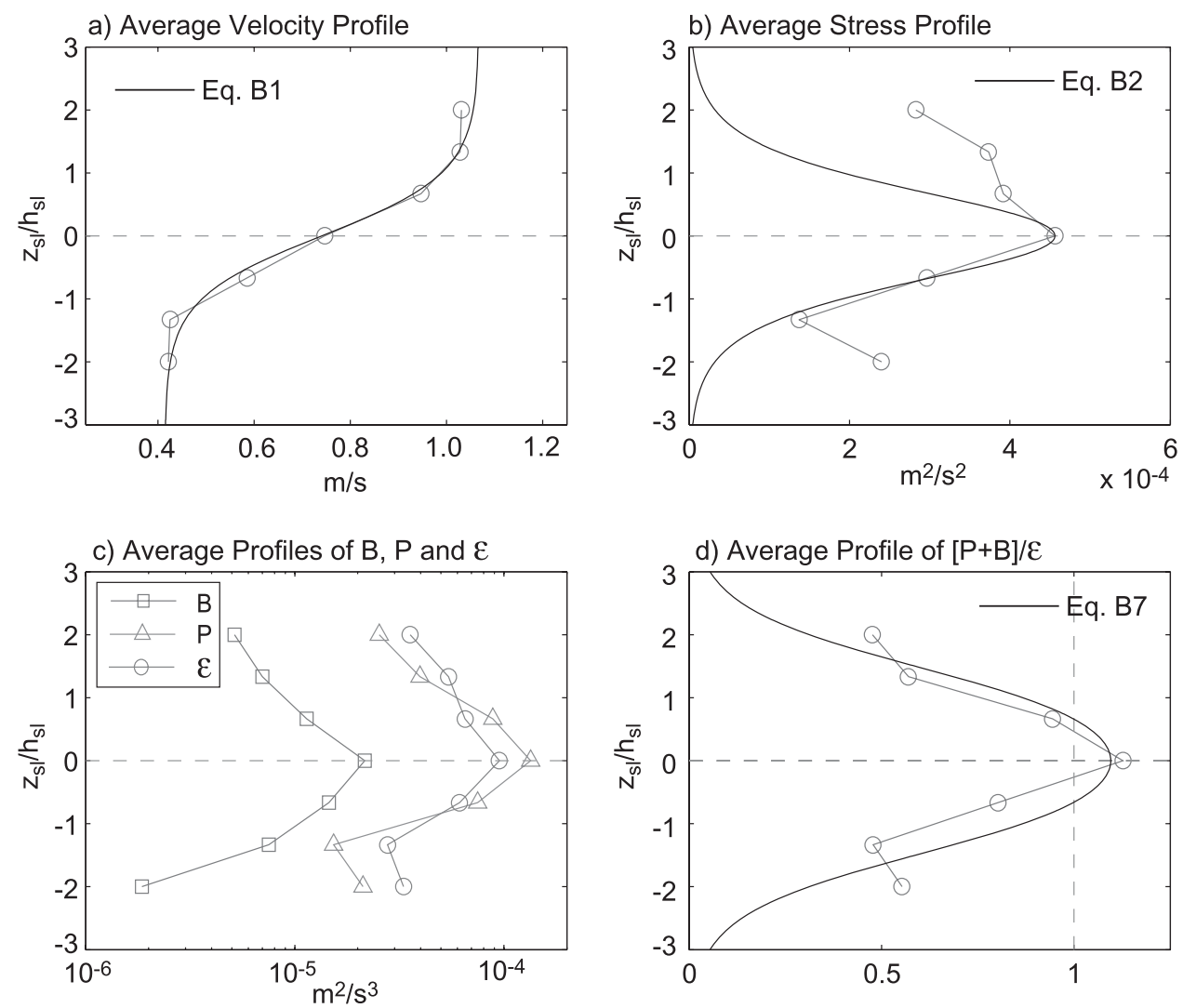

FIG. 12. Average profiles of quantities estimated during Ozmidov-limited shear layer conditions including (a) velocity; (b) stress; (c) buoyancy flux $B$, shear production $P$, and dissipation $\varepsilon$; and (d) the ratio $(P+B) / \varepsilon$. The vertical coordinate system has been transformed so that $z=0$ at the center of the shear layer. All quantities are then averaged over equally spaced intervals of $z_{\mathrm{sl}} / h_{\mathrm{sl}}$, where $h_{\mathrm{sl}}$ is assumed constant and equal to $1.5 \mathrm{~m}$. Only data where $L_{O}<L_{\mathrm{BL}}$ are considered. Solid black lines represent the assumed analytic forms derived in appendix B.

generally consistent with the shear layer scaling that accounts for the divergence in turbulent transport (B7) using the observed average interfacial stress $u_{*}^{2}$ and average velocity difference across the layer $\Delta U$ (Fig. 12d). In applying the scaling, the flux Richardson number $\mathrm{Ri}_{f}$ was assumed to be 0.2 and the thickness of the shear layer was assumed to be $1.5 \mathrm{~m}$.

Consistent with the approach taken for boundary layer conditions, the dissipation estimates for shear layer conditions can be corrected to account for potential influence of the vertical divergence in TKE flux using Eq. (B7). This is done using the measured value of the stress in the interface $\left(z_{\mathrm{s} l} / h_{\mathrm{sl}}=0\right)$ and the total velocity difference measured across the MAST. Consistent with the results presented in Fig. 12d, $\mathrm{Ri}_{f}$ is assumed equal to 0.2 and the width of the shear layer is $1.5 \mathrm{~m}$ and assumed to be constant. Although the value for $h_{\mathrm{sl}}$ most likely increases during the ebb tide, this constant value gives a reasonable fit for the average vertical profiles of velocity and stress (Fig. 12). With estimates of these quantities, the scaling from appendix B is used to reanalyze the TKE balance for shear layer conditions (Fig. 13). As in the boundary layer case, the influence of the divergence in vertical TKE flux is accounted for by multiplying the observed dissipation by the nondimensional ratio given in B7. The uncorrected data show significant deviations from the typically assumed first-order balance of $P+B=\varepsilon$ (slope of logarithmic regression $=0.54 \pm 0.09$; Fig. 13a). In contrast, accounting for the potential influence of vertical TKE flux via Eq. (B7) shows better agreement with the slope of the regression approximately equal to one (slope $=0.96 \pm 0.26$; Fig. 13b). There is more scatter using this approach, which is not surprising given the uncertainty in the quantities needed to apply this scaling. It should be noted that obtaining reliable estimates of direct turbulent fluxes in the strongly stratified center of the shear layer is very challenging because the Ozmidov scale can be smaller than the ADV sampling volume. As a result, the total number of data points in which all the necessary quantities are reliably estimated is relatively small. 

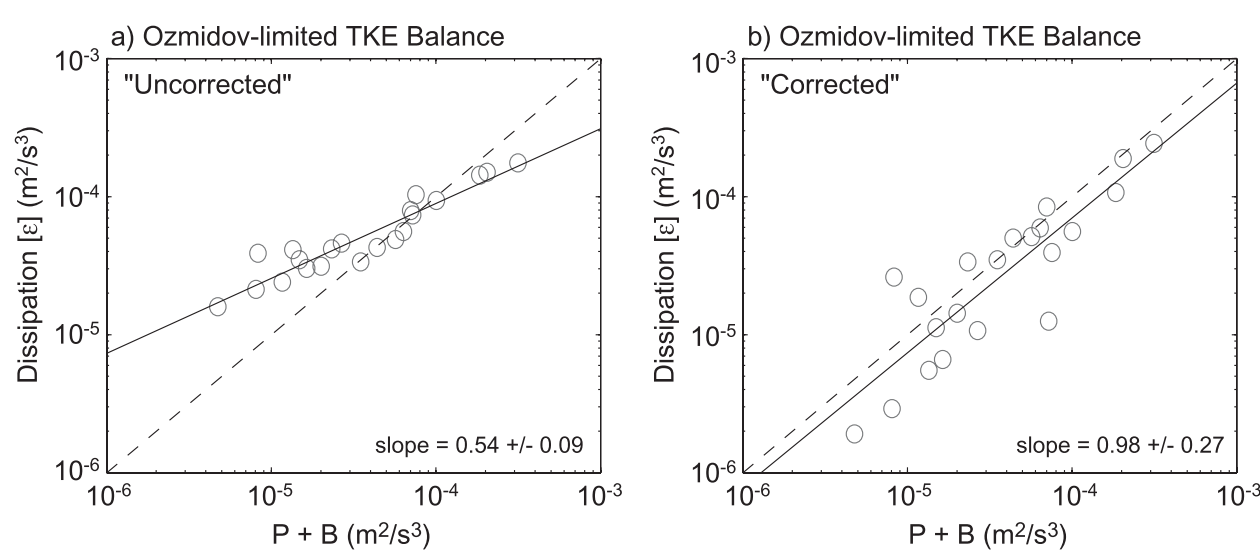

FIG. 13. Estimates of the TKE balance for stratified Ozmidov-limited conditions. The sum of shear production and buoyancy flux is compared to (a) the observed dissipation rate and (b) the corrected dissipation rate after applying a correction for the potential influence of the vertical divergence in turbulent TKE transport [Eq. (B7)]. The solid line is the best-fit least squares regression to the log-transformed data with the regression slope and $95 \%$ confidence interval. The dashed line represents $(P+B)=\varepsilon$. In applying (B7), it is assumed that $\mathrm{Ri}_{f}=0.2$ and $h_{\mathrm{sl}}=1.5 \mathrm{~m}$. Only data where reliable estimates of the interfacial stress were obtained are shown.

\section{f. Nondimensional stress $\left(\left\langle u^{\prime} w^{\prime}\right\rangle / k_{q}\right)$}

In deriving the analytic expressions for the divergence in vertical TKE flux, it is assumed that the ratio of stress to TKE is roughly constant. However, as will be discussed in section $3 \mathrm{~g}$, most turbulence models predict that this ratio should decrease with increasing stratification as well as for conditions when $\varepsilon>P$. Figure $14 \mathrm{a}$ shows the observed ratio $-\left\langle u^{\prime} w^{\prime}\right\rangle / k_{q}$ plot against $P / \varepsilon$ for all data where $\mathrm{Ri}<0.1$. This restriction on $\mathrm{Ri}$ is imposed to remove the influence of stratification and highlight the role of nonlocal turbulent production. Although there is some scatter, the trend clearly demonstrates a reduction in the ratio $-\left\langle u^{\prime} w^{\prime}\right\rangle / k_{q}$ for conditions when $\varepsilon>P$. From the data presented in section $3 \mathrm{e}$, the most likely explanation for conditions where $\varepsilon>P+B$ is the divergence in the vertical flux of TKE. For both boundary layer and shear layer mixing, shear production is greatest where the shear is at its maximum. TKE diffuses away from these regions and is exported to regions of lower shear. Because the stress also is reduced in the lower shear regions, the vertical flux of TKE reduces overall the ratio $-\left\langle u^{\prime} w^{\prime}\right\rangle / k_{q}$.

The presence of density stratification also is expected to reduce the nondimensional stress. To try to isolate the influence of nonlocal turbulent production, the ratio $-\left\langle u^{\prime} w^{\prime}\right\rangle / k_{q}$ is plotted as a function of Ri only for all data where $P+B$ balances $\varepsilon$ to within $20 \%$ (Fig. 14b). These data suggest that there is a general decrease in this ratio as $\mathrm{Ri}$ approaches some critical value. We do not have
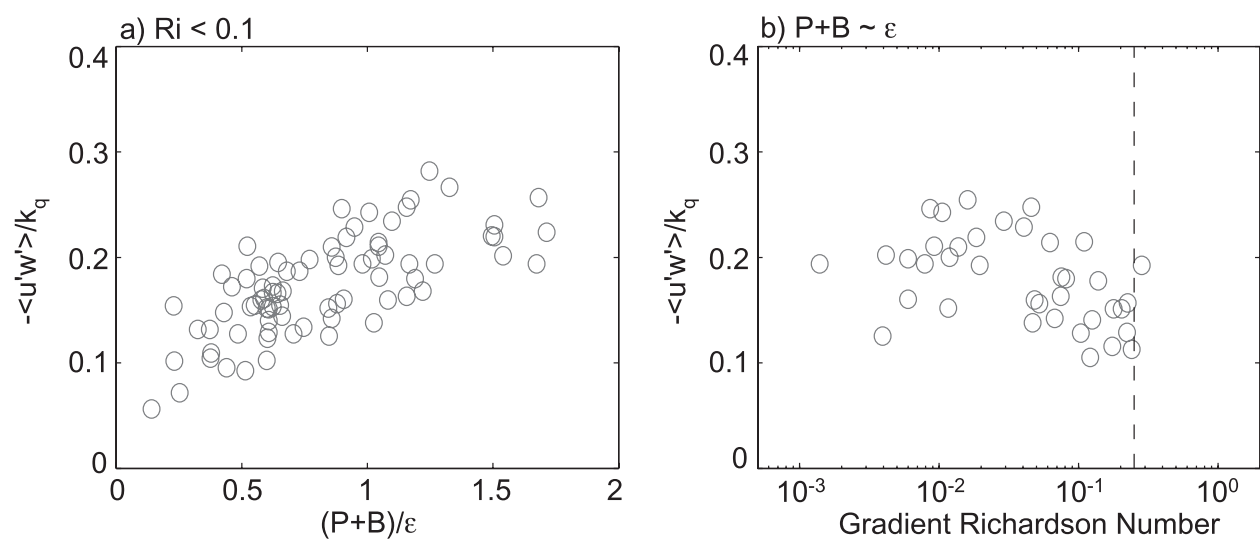

FIG. 14. Comparison of the observed ratio of momentum flux to TKE as a function of (a) deviation from local TKE balance $[(P+B) / \varepsilon]$ for all data where the gradient Richardson number Ri is less than 0.1 and (b) gradient Richardson number for all data where the local TKE balance is satisfied to within $20 \%$. 
a) Constant Stability Function

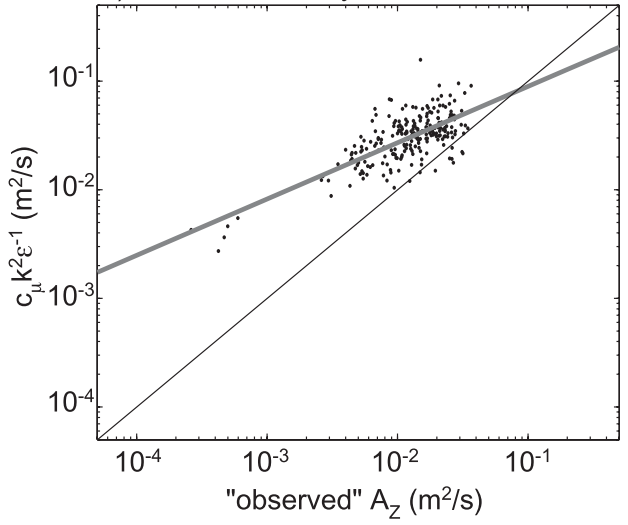

c) Non-Equilibrium (CA 2001)

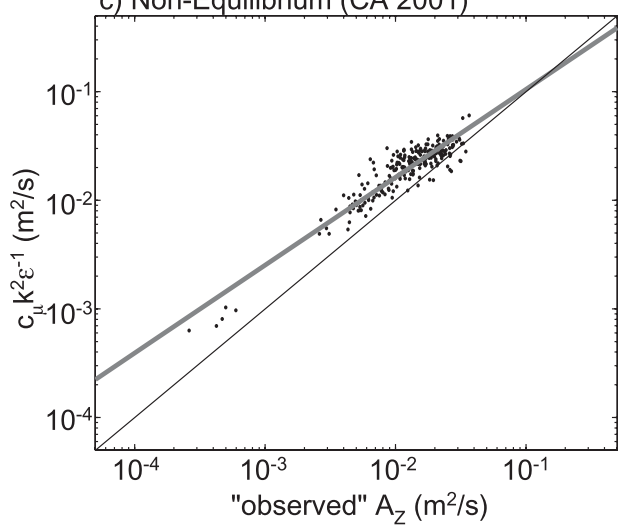

b) Quasi-Equilibrium (KC 1994)

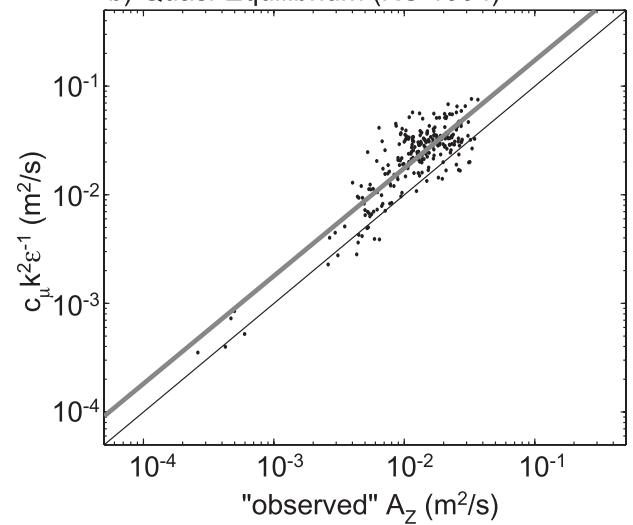

d) Non-Equilibrium (KC 2001)

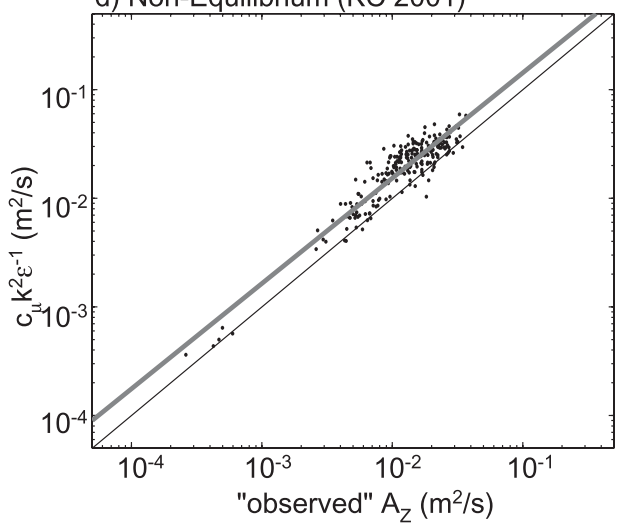

FIG. 15. Comparison of the observed eddy viscosity $A_{z}$ defined as the observed stress divided by the vertical velocity shear to predicted value of $A_{z}$ based on (a) assumed constant value of stability function $\left(c_{\mu}=0.0945\right)$; (b) quasi-equilibrium stability functions of KC94; (c) full equilibrium stability functions of CA01; and (d) full equilibrium stability functions of KC94 as derived in BB01. Solid lines indicate the best-fit least squares regression to the log-transformed data.

any observations where $P+B$ balances $\varepsilon$ to within $20 \%$ for $\mathrm{Ri}>0.29$, generally consistent with linear stability threshold of $\mathrm{Ri}_{\mathrm{cr}}=0.25$. This value also is supported by the increase in anisotropy as $\mathrm{Ri}$ approaches 0.25 (Fig. 7a). However, it is important to note that it becomes increasing difficult to resolve the turbulent quantities presented in this paper as stratification increases and the turbulent length scales decrease. Therefore, we cannot conclusively rule out the possibility of equilibrium mixing for $\mathrm{Ri}>0.25$.

\section{g. Consistency with turbulence closure}

The results presented show boundary layer and shear layer conditions and wide variations in stratification and deviations from a typically assumed TKE balance of $P+$ $B=\varepsilon$. With this range of conditions, these data provide an opportunity to test several commonly used turbulence closure models. Estimates of the momentum flux and vertical shear are used to calculate an observed eddy viscosity [i.e., $A_{z}=-\left\langle u^{\prime} w^{\prime}\right\rangle(\partial u / \partial z)^{-1}$ ], which is compared to values calculated from Eq. (7) using the four different formulations for the stability functions described in section 1c. The two nonequilibrium formulations compare most favorably with the data (Fig. 15). Based on this analysis, the BB01 formulation is slightly better than CA01, because the correlation coefficient is slightly higher and the slope of the logarithmic regression is closer to unity for the BB01 formulation $\left(r^{2}=0.66\right.$ versus 0.68 ; slope $=0.83$ versus 0.99 ). Neither the quasi-equilibrium stability function $\left(r^{2}=0.49\right.$ and slope $\left.=1.05\right)$ nor the constant stability function $\left(r^{2}=0.27\right.$ and slope $\left.=0.55\right)$ compares as favorably as the two nonequilibrium formulations.

Because the only difference between these approaches is the value of the calculated stability functions, it is instructive to directly compare the predicted value of $c_{\mu}$ to its observed value (Fig. 16). The observed value for the stability function is calculated using the observed stress, shear, TKE, and dissipation rate [i.e., $c_{\mu}=$ $\left.-\left\langle u^{\prime} w^{\prime}\right\rangle \varepsilon(\partial u / \partial z)^{-1} k_{q}^{-2}\right]$. The observed values of $c_{\mu}$ vary 

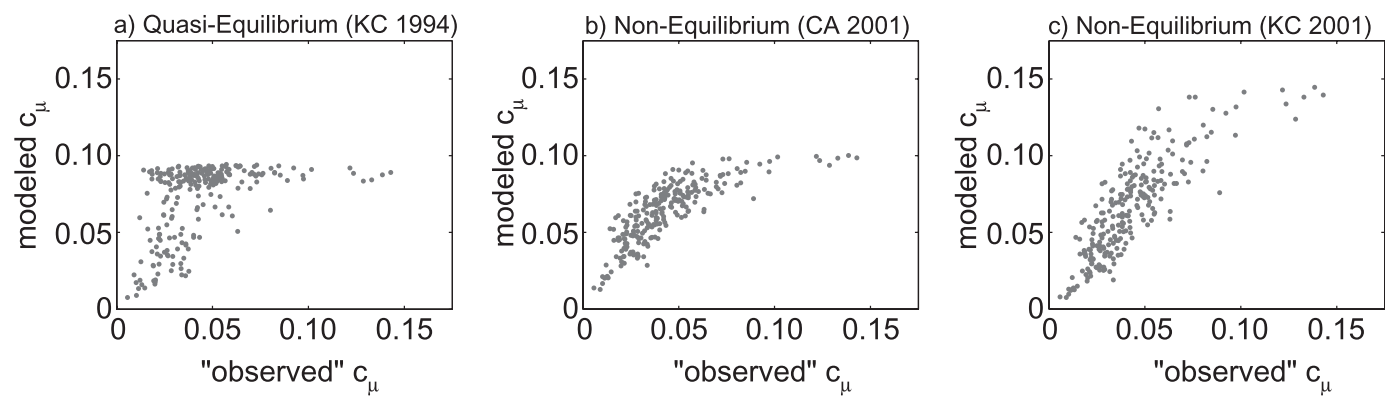

FIG. 16. Comparison of the observed stability function $c_{\mu}$ to that predicted by (a) the quasi-equilibrium stability functions of $\mathrm{KC} 94$; (b) the full equilibrium stability functions of CA01; and (c) the full equilibrium stability functions of $\mathrm{KC} 94$ as derived in BB01. The observed stability function is calculated as the product of observed stress and dissipation rate divided by the product of the vertical velocity shear and TKE squared.

from 0.006 to 0.14 , nearly a factor of 25 . Consistent with the comparison of eddy viscosity, the two nonequilibrium formulations compare most favorably to the observations. The best overall agreement is found for the nonequilibrium model $\mathrm{BB} 01$. Of the three variable stability function parameterizations, all three demonstrate good agreement for low to midrange values of $c_{\mu}$. However, BB01 captures the higher values better than the other two forms. The models of CA01 and KC94 asymptote to maximum values for $c_{\mu}$ of $\sim 0.10$ and $\sim 0.09$, respectively. In contrast, maximum values predicted by BB01 reach $\sim 0.16$. The impact of the lower maximum value of $c_{\mu}$ can be seen in Figs. 16a,b, where the predicted values flatten out as the observed value increases. This behavior is less noticeable for the BB01 model (Fig. 16c). The assumption of a constant stability function is a poor representation of this dataset.

The data presented above show that the nonequilibrium stability functions are more consistent with the data than either a constant stability function or the quasi-equilibrium formulation. One key reason for this is that stability functions in the quasi-equilibrium models are only a function of $\alpha_{N}$. As a result, they asymptote to a constant value of $c_{\mu}$ at low values of $\mathrm{Ri}$, even for conditions when $\varepsilon>P$. In contrast, both nonequilibrium formulations retain their dependence on disequilibrium turbulence at low values of Ri. The most significant difference between the comparisons shown in Fig. 16 is for larger values of $c_{\mu}$, which correspond to low Ri and $\varepsilon \gg P$ in this dataset. It appears that the model of BB01 best represents this dataset, because it allows for greater variability of the stability function for low Ri conditions when dissipation exceeds production.

\section{Discussion and conclusions}

Although the use of the proposed nonequilibrium stability functions significantly improves the comparison between the observed and modeled eddy coefficients, the observed eddy viscosities are roughly $50 \%$ smaller than the modeled values (Fig. 15). Even for unstratified conditions where $\mathrm{P} \approx \varepsilon$, the observed ratio of stress to TKE is roughly $40 \%$ smaller than assumed by turbulence models (Fig. 14a). We cannot conclusively rule out that this is caused by some bias in our methodology. Contamination of our estimates of TKE by nonturbulent motion would explain the observed discrepancies. However, it also is possible that the ratio of momentum flux to TKE is simply lower in an estuarine environment with complex bathymetry than the ratio derived from laboratory experiments.

Despite these discrepancies, we feel that the overall consistency with turbulence models is notable and conclude that the approach used by most second-moment turbulence models captures the key elements of stratified mixing in an estuarine environment. These models are largely consistent the observations presented here because they 1) impose either Ozmidov or boundary layer scaling based on the limiting length scale; 2) account for stratification affects by reducing the ratio of stress to TKE as $\mathrm{Ri}$ approaches $\mathrm{Ri}_{\mathrm{cr}} ; 3$ ) reduce the ratio of stress to TKE for nonlocal turbulence (i.e., $\varepsilon>P+B$ ); and 4) employ a fully dynamic TKE equation that allows for turbulent transport of TKE. Most of these models employ a downgradient formulation for TKE transport, which employs an eddy coefficient that is equal or proportional to the eddy viscosity. This is generally consistent with observations of both boundary layer and shear layer mixing, given the limited data.

Of the formulations considered, the best agreement was found with the nonequilibrium stability functions of KC94 as derived by BB01. This formulation is slightly more consistent than the approach suggested by CA01, because the stability functions are more strongly dependent on deviations from equilibrium turbulence under weakly stratified conditions. Also the data are more 
consistent with $\mathrm{Ri}_{\text {cr }}=0.235$ than the value proposed by CA01 $\left(\mathrm{Ri}_{\mathrm{cr}}=0.847\right)$. However, it should be noted that both of the nonequilibrium stability functions considered perform better than the quasi-equilibrium formulation and that the differences between the nonequilibrium form of KC94 and CA01 are slight. Further, Burchard and Deleersnijder (2001) found that the nonequilibrium form of KC94 was numerically unstable. In many ways the agreement reported here is not surprising given that the parameterizations used in these models are tuned to reproduce the turbulent characteristics of boundary layer and free shear layer flows, the two dominant types of mixing observed here. Similar agreement might not be expected in other environments, where other mixing processes (e.g., surface wave breaking, internal wave energy) contribute significantly to the momentum fluxes.

Acknowledgments. The funding for this research was obtained from ONR Grant N00014-06-1-0292 and NSF Grants and OCE-08-25226 and OCE-08-24871.

\section{APPENDIX A}

\section{Scaling for TKE Flux in a Logarithmic Boundary Layer}

For an unstratified bottom boundary layer, with logarithmic velocity profile and vertical distribution of stress that decays linearly away from the bed to the top of the boundary layer (where stress $\sim 0$ ), shear production can be represented as

$$
P=-\left\langle u^{\prime} w^{\prime}\right\rangle \frac{\partial u}{\partial z}=\frac{u_{*}^{3}}{\kappa z}\left[1-\frac{z}{h_{\mathrm{BL}}}\right],
$$

with an eddy viscosity

$$
A_{z}=u_{*} \kappa z\left[1-\left(z / h_{\mathrm{BL}}\right)\right]
$$

Using the downgradient assumption for the vertical transport of TKE and assuming that the eddy coefficient for the vertical transport of TKE is the same as the eddy viscosity of momentum gives the following form for the vertical flux of TKE:

$$
\left\langle k_{q}^{\prime} w^{\prime}\right\rangle=A_{z} \frac{\partial k_{q}}{\partial z}=-\frac{u_{*}^{3} \kappa}{\left(c_{\mu}^{o}\right)^{2} h}\left[z-\left(\frac{z^{2}}{h}\right)\right]
$$

where the vertical distribution of TKE is simply assumed to be proportional to the stress (via $c_{\mu}^{o}$ ). The divergence in vertical TKE flux is then

$$
\frac{\partial}{\partial z}\left\langle k_{q}^{\prime} w^{\prime}\right\rangle=-\frac{u_{*}^{3} \kappa}{\left(c_{\mu}^{o}\right)^{2} h}\left[1-\left(\frac{2 z}{h}\right)\right] .
$$

Because (A3) depicts a parabolic flux profile, (A4) predicts a linearly varying divergence in flux that is negative in the lower half of the boundary layer (i.e., export of TKE) and positive in the upper half (i.e., import of TKE). Assuming a simple balance between turbulent dissipation, production, and the divergence in turbulent transport gives the following nondimensional function for the ratio of turbulent production to dissipation:

$$
\frac{P}{\varepsilon}=\frac{1-\left(\frac{z}{h}\right)}{\left[1-\left(\frac{z}{h}\right)\right]-\left\{\frac{\kappa^{2}}{\left(c_{\mu}^{o}\right)^{2}}\left[\frac{z}{h}-\left(\frac{2 z^{2}}{h^{2}}\right)\right]\right\}} .
$$

\section{APPENDIX B}

\section{Scaling for TKE Flux in a Stratified Shear Layer}

We next consider mixing associated with a free shear layer where the velocity profile is approximated by a hyperbolic tangent,

$$
u(z)=\frac{\Delta U}{2} \tanh \left(\frac{z_{\mathrm{sl}}}{h_{\mathrm{sl}}}\right)+C,
$$

where $\Delta U$ is the velocity difference across the shear layer, the thickness of the layer is given by $h_{\mathrm{sl}}$, and $C$ is a constant. The vertical distribution of stress can be roughly estimated as

$$
-\left\langle u^{\prime} w^{\prime}\right\rangle=u_{*}^{2} \operatorname{sech}^{2}\left(\frac{z_{\mathrm{sl}}}{h_{\mathrm{sl}}}\right),
$$

where $u_{*}^{2}$ is the scale for the maximum stress at the interface $\left(z_{\mathrm{s} 1} / h_{\mathrm{sl}}=0\right)$. Using the shear associated with velocity profile (B1) and the stress profile given in (B2) results in the following expression for the shear production:

$$
P=-\left\langle u^{\prime} w^{\prime}\right\rangle \frac{\partial u}{\partial z}=\frac{u_{*}^{2} \Delta U}{2 h_{\mathrm{sl}}}\left(\operatorname{sech}^{4} \frac{z_{\mathrm{sl}}}{h_{\mathrm{sl}}}\right) .
$$

The assumed form of the stress distribution and vertical shear gives an eddy viscosity that is constant across the shear layer,

$$
A_{z}=-\frac{\left\langle u^{\prime} w^{\prime}\right\rangle}{\frac{\partial u}{\partial z}}=\frac{2 u_{*}^{2} h_{\mathrm{sl}}}{\Delta U}
$$


Using the same assumptions used to obtain (A3), the vertical flux of TKE can be represented as

$$
\left\langle k_{q}^{\prime} w^{\prime}\right\rangle=-A_{z} \frac{\partial k_{q}}{\partial z}=\frac{4 u_{*}^{4}}{\Delta U\left(c_{\mu}^{o}\right)^{2}} \tanh \left(\frac{z_{\mathrm{sl}}}{h_{\mathrm{sl}}}\right) \operatorname{sech}^{2}\left(\frac{z_{\mathrm{sl}}}{h_{\mathrm{sl}}}\right),
$$

with the divergence in vertical TKE flux given as

$$
\begin{aligned}
-\frac{\partial}{\partial z}\left\langle k_{q}^{\prime} w^{\prime}\right\rangle= & \frac{8 u_{*}^{4}}{\Delta U h\left(c_{\mu}^{o}\right)^{2}}\left[\tanh ^{2}\left(\frac{z_{\mathrm{sl}}}{h_{\mathrm{sl}}}\right) \operatorname{sech}^{2}\left(\frac{z_{\mathrm{sl}}}{h_{\mathrm{sl}}}\right)\right. \\
& \left.-\frac{1}{2} \operatorname{sech}^{4}\left(\frac{z_{\mathrm{sl}}}{h_{\mathrm{sl}}}\right)\right]
\end{aligned}
$$

The flux profile given by $\mathrm{B} 5$ is positive above the interface $\left(z_{\mathrm{sl}} / h_{\mathrm{sl}}>0\right)$ and negative below $\left(z_{\mathrm{sl}} / h_{\mathrm{sl}}<0\right)$, asymptoting to zero as $z_{\mathrm{sl}} / h_{\mathrm{sl}}$ exceeds $\pm \pi$. The expression in B6 results in a negative divergence in TKE flux (i.e., export of TKE) over the central portion of the shear layer $(-0.66<$ $\left.z_{\mathrm{sl}} / h_{\mathrm{sl}}<0.66\right)$ and a positive divergence (i.e., import of TKE) at the upper and lower portions of the shear layer $\left(z_{\mathrm{sl}} / h_{\mathrm{sl}}<-0.66\right.$ and $\left.z_{\mathrm{s}} / h_{\mathrm{sl}}>0.66\right)$.

For the shear layer case, the assumed TKE balance is between turbulent dissipation, shear production, buoyancy flux, and the divergence in turbulent transport. Relating the buoyancy flux term to shear production via the flux Richardson number $\mathrm{Ri}_{f}$ gives the following nondimensional ratio, which accounts for the divergence in turbulent transport:

$$
\frac{P+B}{\varepsilon}=\frac{1-\mathrm{Ri}_{f}}{\left(1-\mathrm{Ri}_{f}\right)+\frac{16 u_{*}^{2}}{\left(c_{\mu}^{o}\right)^{2} \Delta U^{2}}\left[\sinh ^{2}\left(\frac{z_{\mathrm{sl}}}{h_{\mathrm{sl}}}\right)-\frac{1}{2}\right]}
$$

Unlike the expression given in (A5), this expression is a function of several unknown and difficult to estimate quantities, including the stress at the interface, the velocity gradient across the layer, the thickness of the shear layer, and the value of the flux Richardson number. However, despite this more complicated formulation, this scaling can help constrain the overall importance of the divergence in vertical turbulent flux to the observed TKE balance for shear layer mixing.

\section{REFERENCES}

Abarbanel, H. D., D. D. Holm, J. E. Marsden, and T. Ratiu, 1984: Richardson number criterion for the non-linear stability of 3D stratified flow. Phys. Rev. Lett., 52, 2352-2355.

Baumert, H., and H. Peters, 2004: Turbulence closure, steady state, and collapse into waves. J. Phys. Oceanogr., 34, 505-512.
Burchard, H., and K. Bolding, 2001: Comparative analysis of four second-moment turbulence closure models for the oceanic mixed layer. J. Phys. Oceanogr., 31, 1943-1968.

—, and E. Deleersnijder, 2001: Stability of algebraic non-equilibrium second-order closure models. Ocean Modell., 3, 33-50.

— O. Petersen, and T. P. Rippeth, 1998: Comparing the performance of the Mellor-Yamada and the $k-\varepsilon$ two-equation turbulence models. J. Geophys. Res., 103, 10 543-10 554.

Canuto, V. M., A. Howard, Y. Cheng, and M. S. Dubovikov, 2001: Ocean turbulence. Part I: One point closure model-Momentum and heat vertical diffusivities. J. Phys. Oceanogr., 31, 1413-1426.

Dillon, T. M., 1982: Vertical overturns: A comparison of Thorpe and Ozmidov scales. J. Geophys. Res., 87, 9601-9613.

Galperin, B., L. H. Kantha, S. Hassid, and A. Rosati, 1988: A quasiequilibrium turbulent energy model for geophysical flows. J. Atmos. Sci., 45, 55-62.

Gargett, A., T. Osborn, and P. Nasmyth, 1984: Local isotropy and decay of turbulence in a stratified fluid. J. Fluid Mech., 144, 231-280.

Gerbi, G. P., J. H. Trowbridge, J. B. Edson, A. J. Plueddemann, E. A. Terray, and J. J. Fredericks, 2008: Measurements of momentum and heat transfer across the air-sea interface. J. Phys. Oceanogr., 38, 1054-1072.

,-- E. A. Terray, A. J. Plueddemann, and T. Kukulka, 2009: Observations of turbulence in the ocean surface boundary layer: Energetics and transport. J. Phys. Oceanogr., 39, 1077-1096.

Geyer, W. R., M. E. Scully, and D. K. Ralston, 2008: Quantifying vertical mixing in estuaries. Environ. Fluid Mech., 8, 495-509.

Grant, W. D., A. J. Williams III, and S. M. Glenn, 1984: Bottom stress estimates and their prediction on the Northern California continental shelf during CODE-1: The importance of wave-current interaction. J. Phys. Oceanogr., 14, 506-527.

Guo, X., and A. Valle-Levinson, 2008: Wind effects on the lateral structure of density-driven circulation in Chesapeake Bay. Cont. Shelf Res., 28, 2450-2471.

Hossain, M. S., 1980: Mathematische Modellierung von turbulentn Auftriebsstomungen. Ph.D. dissertation, University of Karlsruhe, 145 pp.

Howard, L. N., 1961: Note on a paper of John W. Miles. J. Fluid Mech., 10, 509-512.

Kaimal, J. C., J. C. Wyngaard, Y. Izumi, and O. R. Cote, 1972: Spectral characteristics of surface-layer turbulence. Quart. J. Roy. Meteor. Soc., 98, 563-589.

Kantha, L. H., and C. A. Clayson, 1994: An improved mixed layer model for geophysical applications. J. Geophys. Res., 99, $25235-25266$.

Li, M., L. Zhong, and W. C. Boicourt, 2005: Simulations of Chesapeake Bay estuary: Sensitivity to turbulence mixing parameterizations and comparison with observations. J. Geophys. Res., 110, C12004, doi:10.1029/2004JC002585.

,,$-- \ldots$, S. Zhang, and D.-L. Zhang, 2006: Hurricaneinduced storm surges, currents and destratification in a semienclosed bay. Geophys. Res. Lett., 33, L02604, doi:10.1029/ 2005GL024992.

Lumley, J. L., and E. A. Terray, 1983: Kinematics of turbulence convected by a random wave field. J. Phys. Oceanogr., 13, 2000-2007.

Mauritsen, T., and G. Svensson, 2007: Observations of stably stratified shear-driven atmospheric turbulence at low and high Richardson numbers. J. Atmos. Sci., 64, 645-655.

Mellor, G., and T. Yamada, 1982: Development of a turbulence closure model for geophysical fluid problems. Rev. Geophys., 20, 851-875. 
Miles, J. W., 1961: On the stability of heterogeneous shear flows. J. Fluid Mech., 10, 496-508.

Peters, H., and H. Z. Baumert, 2007: Validating a turbulence closure against estuarine microstructure measurements. Ocean Modell., 19, 183-203.

Ralston, D. K., W. R. Geyer, and J. A. Lerczak, 2010a: Structure, variability, and salt flux in a strongly forced salt wedge estuary. J. Geophys. Res., 115, C06005, doi:10.1029/2009JC005806.

,,--- , and M. E. Scully, 2010b: Turbulent mixing in a strongly forced salt wedge estuary. J. Geophys. Res., 115, C12024, doi:10.1029/2009JC006061.

Rodi, W., 1980: Turbulence Models and Their Application to Hydraulics. International Association for Hydraulic Research, 104 pp.

Scully, M. E., 2010: Wind modulation of dissolved oxygen in Chesapeake Bay. Estuaries Coasts, 33, 1164-1175.

_ W. R. Geyer, and J. A. Lerczack, 2009: The influence of lateral advection on the residual circulation: A numerical modeling study of the Hudson River estuary. J. Phys. Oceanogr., 39, 107-124.

Simpson, J. H., H. Burchard, N. R. Fisher, and T. P. Rippeth, 2002: The semi-diurnal cycle of dissipation in a ROFI: Modelmeasurement comparisons. Cont. Shelf Res., 22, 1615-1628.
Smyth, W. D., J. N. Moum, and D. R. Caldwell, 2001: The efficiency of mixing in turbulent patches: Inferences from direct numerical simulation and microstructure observations. J. Phys. Oceanogr., 31, 1969-1992.

Stacey, M. T., S. G. Monismith, and J. R. Burau, 1999: Observations of turbulence in a partially stratified estuary. J. Phys. Oceanogr., 29, 1950-1970.

Tennekes, H., and J. L. Lumley, 1972: A First Course in Turbulence. MIT Press, $300 \mathrm{pp}$.

Trowbridge, J., and S. Elgar, 2003: Spatial scales of stress-carrying nearshore turbulence. J. Phys. Oceanogr., 33, 1122-1128.

Turner, J. S., 1973: Buoyancy Effects in Fluids. Cambridge University Press, 369 pp.

Umlauf, L., and H. Burchard, 2003: A generic length-scale equation for geophysical turbulence models. J. Mar. Res., 61, $235-265$.

Voulgaris, G., and J. H. Trowbridge, 1998: Evaluation of the acoustic Doppler velocimeter (ADV) for turbulence measurements. J. Atmos. Oceanic Technol., 15, 272-289.

Warner, J. C., W. R. Geyer, and J. A. Lerczak, 2005: Numerical modeling of an estuary: A comprehensive skill assessment. J. Geophys. Res., 110, C05001, doi:10.1029/2004JC002691. 